\title{
Effects of heat release in a turbulent, reacting shear layer
}

\author{
By J. C. HERMANSON† AND P. E. DIMOTAKIS \\ Graduate Aeronautical Laboratories, California Institute of Technology, \\ Pasadena, CA 91125, USA
}

(Received 24 July 1987 and in revised form 21 April 1988)

Experiments were conducted to study the effects of heat release in a planar, gas-phase, reacting mixing layer formed between two free streams, one containing hydrogen in an inert diluent, the other, fluorine in an inert diluent. Sufficiently high concentrations of reactants were utilized to produce adiabatic flame temperature rises of up to $940 \mathrm{~K}$ (corresponding to $1240 \mathrm{~K}$ absolute). The temperature field was measured at eight fixed points across the layer. Flow visualization was accomplished by schlieren spark and motion picture photography. Mean velocity information was extracted from Pitot-probe dynamic pressure measurements. The results showed that the growth rate of the layer, for conditions of zero streamwise pressure gradient, decreased slightly with increasing heat release. The overall entrainment into the layer was substantially reduced as a consequence of heat release. A posteriori calculations suggest that the decrease in layer growth rate is consistent with a corresponding reduction in turbulent shear stress. Large-scale coherent structures were observed at all levels of heat release in this investigation. The mean structure spacing decreased with increasing temperature. This decrease was more than the corresponding decrease in shear-layer growth rate, and suggests that the mechanisms of vortex amalgamation are, in some manner, inhibited by heat release. The mean temperature rise profiles; normalized by the adiabatic flame temperature rise, were not greatly changed in shape over the range of heat release of this investigation. A small decrease in normalized mean temperature rise with heat release was however observed. Imposition of a favourable pressure gradient in a mixing layer with heat release resulted in an additional decrease in layer growth rate, and caused only a very slight increase in the mixing and amount of chemical product formation. The additional decrease in layer growth rate is shown to be accounted for in terms of the change in free-stream velocity ratio induced by the pressure gradient.

\section{Introduction}

Combustion processes in turbulent flows are of great interest and are significant in many practical applications. Because chemical reactions can occur only when the reactants become molecularly mixed, understanding the flow processes leading to mixing is vital to an understanding of turbulent combustion. Alternatively, the amount and location of chemical product formation can provide an important 'window' into the fluid mechanics of combustion. The amount of chemical product formed as a result of mixing cannot exceed the amount of reactant species entrained into the layer, and the entrainment itself is related to the dynamics of the turbulent flow structure.

† Presently at United Technologies Research Center, East Hartford, CT 06108. 
A major advance in understanding turbulent shear flow was the discovery that important aspects of the flow processes in a shear layer are dominated by large-scale organized structures. This was reported by Brown \& Roshko $(1971,1974)$, Winant $\&$ Browand (1974), Dimotakis \& Brown (1976), and others. The discovery of largescale organization has motivated new measurements of composition. Konrad (1976) measured the probability density function of the concentration of individual species in a non-reacting gas-phase layer. Those results allowed an estimation of the amount of product that would be obtained in a chemically reacting shear layer. Another significant finding reported in that work was an asymmetry in the shear-layer entrainment, with more high-speed than low-speed fluid entrained. Detailed concentration measurements were performed in a liquid-phase shear layer by Koochesfahani \& Dimotakis (1986). Those measurements affirmed that species transport in the layer is dominated by large-scale structures. It was also shown that, at high Reynolds numbers, the composition of the mixed fluid is quite uniform within individual structures.

The amount of mixing and product formation in a gas-phase shear flow with chemical reaction was studied by Mungal \& Dimotakis (1984), utilizing the hydrogen-fluorine reaction. The effects of Reynolds number and chemical reaction rate were addressed in the same facility by Mungal, Hermanson \& Dimotakis (1985) and Mungal \& Frieler (1985), respectively. In addition, the structure of a gas-phase chemically reacting mixing layer prior to the mixing transition was examined by Masutani \& Bowman (1986) using the nitric oxide-ozone reaction. Those investigations were all conducted under conditions of low heat release, with no apparent coupling between heat release and the fluid mechanics. Exploratory studies of reacting shear layers in liquid were performed by Dimotakis \& Brown (1976), more systematically by Breidenthal (1981), who determined the integral amount of product in the layer, and by Koochesfahani \& Dimotakis (1986), who were able to provide detailed measurements of product concentration and its average profile across the layer.

Much of the recent research in flows with combustion has been directed toward understanding the effects of the flow field on the combustion process. These considerations have led to the development of analytical models - for example, the strained-flame models of Marble \& Broadwell (1977) and Broadwell \& Breidenthal (1982), and models for the probability density function of a passive scalar proposed by Pope (1981), Kollman \& Janicka (1982), and Effelsberg \& Peters (1983). Little research effort has been directed, however, at the reverse problem: understanding how the combustion processes affect the flow field, and, in particular, the layer growth, entrainment, and large-scale structure dynamics. The study of these effects was the primary focus of the present investigation.

An exploratory investigation into the effects of heat release was conducted by Wallace (1981), who used the nitric oxide-ozone reaction to study the mixing and combustion in a reacting shear layer. The maximum adiabatic flame temperature rise attained in that investigation was about $400 \mathrm{~K}$. Studies in reacting shear layers at high temperatures $(\approx 1400 \mathrm{~K}$ flame temperature rise) were performed by Ganji $\&$ Sawyer (1980), Keller \& Daily (1983) and Pitz \& Daily (1983).

The present work is a sequel to the experiments of Mungal \& Dimotakis (1984) and can be considered to fill the gap between the low-heat-release studies of Mungal \& Dimotakis (1984), and Wallace (1981), and the high-heat-release investigations of Ganji \& Sawyer (1980), Keller \& Daily (1983) and Pitz \& Dailey (1983). The reactants employed in the present experiments, hydrogen and fluorine, allowed the 
systematic study of heat release effects over a wide range of temperatures. In this work the adiabatic flame temperature rise ranged from $186 \mathrm{~K}$ to $940 \mathrm{~K}$, placing the adiabatic flame temperature rise normalized by the ambient temperature in the range $0.62 \leqslant \Delta T_{\mathrm{f}} / T_{0} \leqslant 3.1$.

Heat release might be expected to alter both the macroscopic properties of a mixing layer (such as the layer growth, entrainment and large-scale structure dynamics) and the microscopic layer properties (for example, the mixing and the amount of chemical product formation). It is not obvious a priori, for example, whether the release of heat by a reacting flow would serve to increase or decrease the rate of growth and entrainment of a shear layer. One might expect that the wedge-like geometry of a shear layer would result in an increased rate of growth owing to the dilatation and consequent displacement effects caused by an increase in temperature in the mixing zone. The earlier results of Wallace (1981), however, suggested a slight decrease in layer growth rate with increasing heat release. Wallace (1981) also found some decrease in layer entrainment with heat release. An interesting, related question is what effect heat release might have on the entrainment ratio.

The amount of mixing that occurs in a shear layer can be measured or inferred by means of various techniques. Two methods which have been employed are the socalled passive-scalar technique and the chemical-reaction technique. In the passivescalar technique, the concentration of a non-reacting entity serving as a conserved scalar in the fluid (e.g. dye or a selected species) is measured and then used to determine the amount of mixing (Rebollo 1973 and Konrad 1976 in gases; Koochesfahani \& Dimotakis 1986 in liquids). The chemical-reaction method relies on the molecular mixing to allow the formation of some sort of product, which once measured is used to infer the amount of molecular mixing that occurred (in gas, Wallace 1981, Mungal \& Dimotakis 1984 and Masutani \& Bowman 1986; in liquid Breidenthal 1981 and Koochesfahani \& Dimotakis 1986). The present work used the chemical-reaction technique, with the measured temperature rise (heat release) used to determine the amount of product formed.

The effects of pressure gradient in reacting flow are of interest because many practical devices have fixed geometry and do not have a means of manipulating the pressure gradient. Some investigators, for example Bray \& Libby (1981) and Spalding (1986), have suggested that a pressure gradient might serve to enhance the mixing and combustion in a reacting flow. The effects of pressure gradient on layer growth and flow structure were investigated by Rebollo (1973) in a non-reacting shear layer. In some cases reported in the present work, a favourable pressure gradient was imposed on the flow, and the layer growth and product formation were also investigated under these conditions.

The experimental facility and measuring techniques will be discussed in $\$ 2$. Sections 3 and 4 contain discussions on the chemistry and run conditions. The effects of heat release on the growth and entrainment of the layer are examined in $\$ 5$. The amount of product formation and the large-scale structure dynamics are discussed in $\$ \S 6$ and 7 , respectively. Finally, the effects of a favourable pressure gradient on the growth and product formation of a reacting mixing layer are presented in $\S 8$.

\section{Experimental facility and instrumentation}

\subsection{Flow apparatus}

The experiments described here were conducted in the blow-down facility documented by Mungal \& Dimotakis (1984), which was partly modified as described 

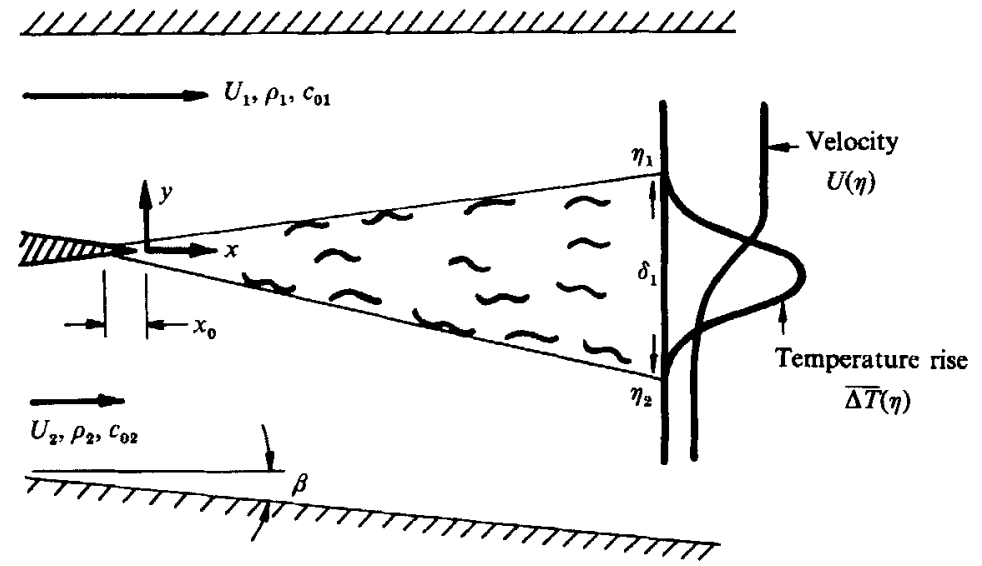

Figure 1. Turbulent shear-layer geometry. $\eta \equiv y /\left(x-x_{0}\right)$.

by Hermanson (1985). Specifically, a planar shear flow was established between free streams resulting from the discharge of separate mixtures of fluorine in an inert diluent, and hydrogen premixed with a small amount of nitric oxide in an inert diluent. The densities of the free streams were matched for most cases by using a gas mixture consisting of nitrogen and a small amount of helium, on the fluorine side, and a mixture of nitrogen and a small amount of argon on the hydrogen side. This procedure was also used to match the heat capacities of the two free streams, allowing the measured temperature rise to be simply related to the heat release in the flow. The high-speed stream emerged from a 6:1 contraction with an exit area of $5 \times 20 \mathrm{~cm}$, and the low-speed stream from a $4: 1$ contraction with a $7.5 \times 20 \mathrm{~cm}$ exit area. The gas mixtures were discharged into the contraction section through sonic orifices. Small adjustments in throat areas were made to minimize variations in freestream velocity from run to run which would have resulted from the differences in gas properties of the various mixtures employed. The two streams met at the tip of a horizontal stainless steel splitter plate with an included angle of $3.8^{\circ}$. The test section geometry is shown schematically in figure 1.

The high-speed upper wall was fixed for all runs at $0^{\circ}$ (horizontal). The divergence angle of the low-speed lower wall was adjusted before each run for the desired streamwise pressure gradient. The wedge-like geometry of the planar shear-layer displacement allows this simple means of accommodating or imposing a pressure gradient. Most of the runs in the present investigation were performed with the upper and lower walls adjusted to the requisite divergence angle to ensure a zero streamwise pressure gradient. For some runs, the walls were left fixed at the angle required for zero pressure gradient at zero heat release, which allowed the heat release to induce a favourable streamwise pressure gradient (accelerating flow), as a result of the combustion displacement effects.

\subsection{Instrumentation}

Five diagnostic techniques were employed in this investigation: schlieren/ shadowgraph flow visualization, cold-wire resistance thermometer and thermocouple temperature measurement, Pitot-probe measurements of the mean dynamic pressure transverse profiles and measurements of two-point streamwise pressure gradient.

High-time-resolution spark schlieren photographs were taken with a spark source ( $\approx 3 \mu$ s duration), synchronized with a motor-driven $35 \mathrm{~mm}$ camera, at a rate of 
approximately three frames per second. Motion picture data were taken with a highspeed pin register camera (Teledyne Model DBS 55) at a rate of 450 frames per second. For the latter, continuous illumination was provided by a $200 \mathrm{~W}$ mercury vapour lamp. The schlieren method is more sensitive than the shadowgraph technique and is better suited for low-heat-release runs. It was found for the conditions of this investigation, even at the higher flow temperatures, that the schlieren technique resulted in better large-scale structure resolution than the shadowgraph method. A circular source mask and a circular hole spatial filter were used in place of the conventional source slit and knife edge in an effort to give equal weights to gradients in index of refraction in all directions and thus better resolve the large-scale vortical structure of the flow. The hole sizes were increased with increasing flow temperature to adjust the sensitivity as necessary.

Temperature data were recorded with a rake of eight $2.5 \mu \mathrm{m}$ diameter platinum-10\% rhodium cold wires, with a wire span of $1-1.5 \mathrm{~mm}$, welded to sharpened Inconel prongs of 0.014 in. $(0.36 \mathrm{~mm})$ diameter. In the highly corrosive environment of these experiments, it was found that the $2.5 \mu \mathrm{m}$ resistance wires in the hottest regions did not survive runs in which the adiabatic flame temperature rise exceeded approximately $600 \mathrm{~K}$ (corresponding to $900 \mathrm{~K}$ absolute). For this reason, a rake of $25 \mu \mathrm{m}$ Chromel-Alumel thermocouples was employed for the hottest runs. The leads of the $25 \mu \mathrm{m}$ thermocouple junctions (Omega Engineering CHAL-001) were welded to prongs consisting of Chromel and Alumel wires of $0.010 \mathrm{in}$. $(250 \mu \mathrm{m})$ diameter. Both the cold wire and thermocouple rakes were positioned across the transverse extent of the layer. The probes were equally spaced at nominal intervals of $1 \mathrm{~cm}$, which sufficed to capture the mean temperature profile. The total data rate for the resistance wires was $80 \mathrm{kHz}$, corresponding to $10 \mathrm{kHz}$ per probe. This rate was consistent with the estimated frequency response of the wires (see Mungal \& Dimotakis 1984). From that work it can be concluded that the characteristic thickness of the reaction zones (flame sheets) as well as the Kolmogorov scales (calculated using either a hot or cold value of kinematic viscosity) were beyond the resolution capabilities of the resistance wire probes. The thermocouples were sampled at $500 \mathrm{~Hz}$ each, for a total data rate of $4 \mathrm{kHz}$; their considerably lower frequency response did not warrant a higher rate.

Thermocouples normally do not require calibration. The resistance wires were calibrated as described in Mungal et al. (1985) using hot and cold jets of known temperature, yielding calibration constants to convert voltage to temperature rise. An additional correction was applied to the output signal voltage in the present experiments to account for the nonlinearity (quadratic coefficient) in the resistivity of the platinum-10\% rhodium wire element at elevated temperatures (see Caldwell 1962). Using methods presented in Scadron \& Warshawsky (1952), it was determined that for neither the thermocouple nor the resistance wires was there significant radiation error for the temperatures in this investigation. Conduction to the support prongs could, however, have resulted in excursions from the mean temperature being in error (low) by as much as $10 \%$ to $20 \%$ for the cold wires and up to $40 \%$ for the thermocouples. Both diagnostics nonetheless produced accurate mean temperatures, as during a small fraction of the course of the run (before data acquisition began) the tips of the support prongs equilibrated to the local mean value. Good agreement (typically within $5 \%$ ) was obtained in runs in which both sets of probes were employed. Errors resulting from differences in the thermal conductivities between the free streams were small (Hermanson 1985).

The mean velocity was extracted from the mean dynamic pressure profile, which 
was measured by a Pitot probe rake of 15 probes connected to a miniature manometer bank filled with a fluorine resistant oil (Hooker Chemical Fluorolube FS5). The bank had an adequate time response to yield a reliable mean dynamic pressure profile during each run. The bank was photographed by a second motordriven $35 \mathrm{~mm}$ camera. The photographic data were digitized using a HewlettPackard HP9874 digitizer and processed to yield dynamic pressure profiles. This technique of measuring the Pitot pressure was estimated to be accurate to approximately $5 \%$. Rebollo (1973) estimated that the accuracy of extracting mean velocities from Pitot and test-section wall static pressures in a flow with a free-stream density ratio of $\rho_{1} / \rho_{2}=7$ is about $4-5 \%$. In the present experiment, the density ratio of the cold free streams to the hot layer centre is at most three, suggesting that the Rebollo error estimate represents an upper bound under these conditions.

Streamwise pressure gradient information was obtained from pressure taps on the low-speed lower wall. The static pressure was measured at two downstream locations $(x=5.1 \mathrm{~cm}, x=30.0 \mathrm{~cm})$ and recorded as a differential using a Datametries type 573 fluorine-resistant Barocel sensor.

\section{Chemistry}

The chemical kinetics of the hydrogen-fluorine system are summarized in Mungal $\&$ Dimotakis (1984). The reaction yields a temperature rise of $93 \mathrm{~K}$ for $1 \% \mathrm{~F}_{2}$ and $1 \% \mathrm{H}_{2}$ in $\mathrm{N}_{2}$ diluent under constant-pressure, adiabatic conditions (this is the socalled adiabatic flame temperature rise). Proper chain initiation requires some free $\mathrm{F}$ atoms, which were generated in these experiments by premixing a trace amount $(3 \%$ of the free-stream $\mathrm{F}_{2}$ concentration) of nitric oxide into the hydrogen-carrying stream.

For all flows reported here, the resulting chemical timescales were fast compared with the fluid mechanical timescales. The chemical timescales for the reaction, over the entire range of concentrations, were estimated using the CHEMkin chemical kinetics program developed by Kee, Miller \& Jefferson (1980). The chemical rate data for the reactions involved were taken from Cohen \& Bott (1982) and Baulch et al. (1981). The Damköhler number (ratio of mixing time to chemical time) based on the local, large-seale characteristic time $\left(\delta_{1} / \Delta U\right.$, where $\delta_{1}$ is the width indicated by the $1 \%$ level of the mean temperature profile and $\Delta U=U_{1}-U_{2}$ is the free-stream velocity difference) ranged from 25 to 130 with increasing reactant concentrations. The work of Mungal \& Frieler (1985) suggests that the chemistry can be regarded as being fast when the Damköhler number for the large seales exceeds 10. A threshold Damköhler number of 10 for the large scales is also suggested by the results of Masutani \& Bowman (1986). Chemical kinetics are, consequently, not an issue in the present investigation, where the chemistry was much faster as a result of the higher reactant concentrations and combustion temperatures.

\section{Run conditions}

The nominal flow velocities for this investigation were

$$
U_{1}=22 \mathrm{~m} / \mathrm{s}, \quad U_{2}=8.8 \mathrm{~m} / \mathrm{s}, \quad r \equiv U_{2} / U_{1}=0.40,
$$

with nominal equal free-stream densities, i.e.

at one atmosphere pressure.

$$
s \equiv \frac{\rho_{2}}{\rho_{1}}=1,
$$




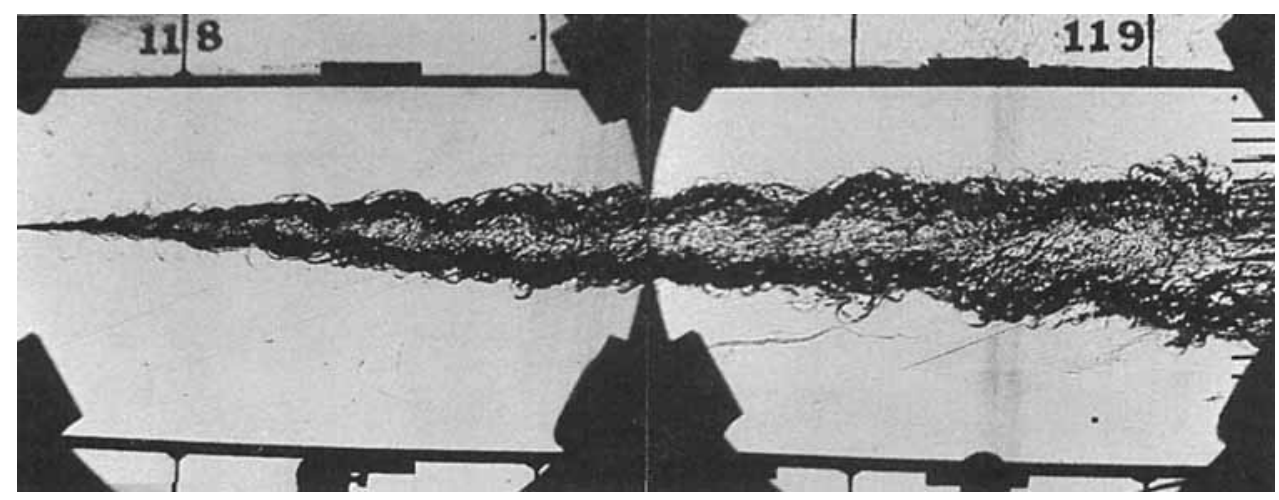

Figure 2. Composite spark schlieren photograph. Flow is from left to right. Top stream : $5 \%$ $\mathrm{H}_{2}, U_{1}=22 \mathrm{~m} / \mathrm{s} ;$ lower stream : $5 \% \mathrm{~F}_{2}, U_{2}=8.8 \mathrm{~m} / \mathrm{s} . \Delta T_{\mathrm{f}}=457 \mathrm{~K} ; R e_{\delta_{1}}=6.2 \times 10^{4}$.

Reactant concentrations ranged from $2 \%$ fluorine and $2 \%$ hydrogen up to $6 \%$ fluorine and $24 \%$ hydrogen, corresponding to an adiabatic flame temperature rise in the range

$$
186 \mathrm{~K} \leqslant \Delta T_{\mathrm{f}} \leqslant 940 \mathrm{~K}
$$

resulting in a maximum local mean temperature rise in the range

$$
125 \mathrm{~K} \leqslant \overline{\Delta T}_{\max } \leqslant 550 \mathrm{~K} \text {. }
$$

The density changes corresponding to the adiabatic flame temperature rise were

$$
0.38 \leqslant \frac{\Delta \rho_{\mathrm{f}}}{\rho_{0}} \leqslant 0.76,
$$

where $\Delta \rho_{\mathrm{p}}$ is the density difference between the cold free streams and the density in the layer, calculated using the adiabatic flame temperature rise and neglecting small differences in static pressure in the layer. The corresponding density changes calculated using the maximum local mean temperature rise were

$$
0.29 \leqslant \frac{\Delta \rho_{\max }}{\rho_{0}} \leqslant 0.65 .
$$

A composite spark schlieren photograph of two different runs is shown in figure 2 . The flow is from left to right with the high-speed free stream in the upper half of the figure. The splitter plate tip and the temperature and Pitot probe rakes at the measuring station are visible at the extreme left and right edges of the figure, respectively.

The local Reynolds number at the measuring station was typically

$$
R e_{\delta_{1}} \equiv \Delta U \delta_{1} / \nu \approx 6 \times 10^{4},
$$

where $\nu$ is the cold free-stream kinematic viscosity. This value of the Reynolds number is well above that for the mixing transition as reported by Konrad (1976), Breidenthal (1981) and Bernal et al. (1979). Mungal \& Dimotakis (1984) and Koochesfahani \& Dimotakis (1986) report that the width $\delta_{1}$ correlates well with the layer visual thickness $\delta_{\text {vis }}$ as defined by Brown \& Roshko (1974); this was also confirmed in the present study. The measuring station for the cold wires and Pitot was at $x=45.7 \mathrm{~cm}$ downstream of the splitter plate trailing edge for all runs. The 
thermocouple rake was normally positioned at $x=44.8 \mathrm{~cm}$. The corresponding Reynolds number based on the high-speed free-stream velocity and on the downstream distance was typically $\operatorname{Re}_{x} \equiv U_{1} x / \nu \approx 6 \times 10^{5}$. The momentum thickness of the high-speed boundary layer on the splitter plate, $\theta_{1}$, was calculated by Thwaite's method and yielded a Reynolds number based on momentum thickness of $U_{1} \theta_{1} / \nu \approx 240$. At the measuring station the normalized distance to the splitter-plate tip was estimated to be $x / \theta_{1} \approx 2800$. This is substantially larger than the value of $x / \theta_{1}=1000$ which Bradshaw (1966) suggests may be required for the layer to become self-similar. This value of $x / \theta_{1}$ is in the range of other shear-layer investigations, for example $x / \theta_{1} \approx 2300$ in Dimotakis \& Brown (1976), $x / \theta_{1} \approx 2900$ in Browand \& Latigo (1979), and $x / \theta_{1} \approx 4000$ for Brown \& Roshko (1974).

The stoichiometric mixture ratio, $\phi$, is defined here as the ratio of the low-speed free-stream molar concentration, $c_{02}$, to the high-speed free-stream molar concentration, $c_{01}$, divided by the low-speed to high-speed molar stoichiometric ratio:

$$
\begin{gathered}
\phi \equiv \frac{\left(c_{02} / c_{01}\right)}{\left(c_{02} / c_{01}\right)_{\mathrm{s}}}, \\
\phi=\frac{c_{02}}{c_{01}},
\end{gathered}
$$

since the hydrogen-fluorine stoichiometric ratio is unity. Equations (1) and (2) can be regarded as representing the volume (or mass) of high-speed free-stream fluid necessary to react completely with a unit volume (or mass) of low-speed free-stream fluid. Volume and mass, if differential diffusion effects are ignored, are interchangeable in this discussion because the free-stream densities in this experiment were nominally equal. For this investigation, the stoichiometric mixture ratio was in the range $\phi=1$ to $\phi=\frac{1}{8}$, with the lean reactant, fluorine, always on the low-speed side of the layer. As the heat release was increased, it was determined that no obvious deviation from the simple trends with heat release (to be discussed later) warranted extending the heat release further. This consideration, in addition to issues of safety, restricted the use of high-speed free-stream hydrogen concentrations to a maximum of $24 \%$. As a consequence, for the higher levels of heat release the extreme value of stoichiometric mixture ratio was $\phi=\frac{1}{4}$.

All the flows in the present investigation were momentum-dominated with negligible effects of buoyancy. For the present investigation, the maximum value of the Richardson number is

$$
R i \approx \frac{\Delta \rho}{\rho_{0}} \frac{g \delta_{1}}{(\Delta U)^{2}} \approx 0.004,
$$

where $g$ is the gravitational constant. Koop \& Browand (1979) suggest that a minimum value of $R i \approx 0.05$ is required in a shear layer for the effects of buoyancy to become important.

\section{Shear-layer growth and entrainment}

\subsection{Shear-layer growth rate}

The thickness of the shear layer, as indicated by the width of the mean temperature profile at a fixed downstream location, decreased slightly with increasing heat release. The observed $1 \%$ temperature profile thickness at zero pressure gradient, normalized by the downstream distance $x-x_{0}$ (here taken to be constant), is plotted 


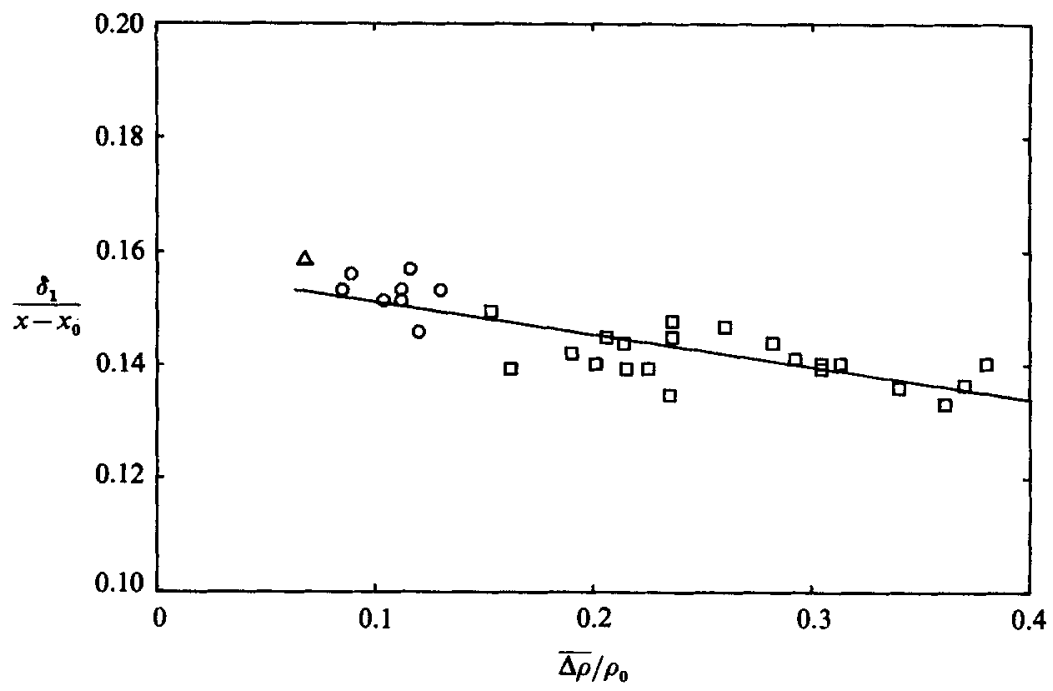

Figure 3. Temperature profile thickness growth rate vs. heat release. $\triangle$, Wallace (1981); O, Mungal \& Dimotakis (1984); $\square$, present results.

in figure 3 versus the mean density reduction parameter, $\overline{\Delta \rho} / \rho_{0}$. Results obtained from Mungal \& Dimotakis (1984) and Wallace (1981) are also presented. The parameter $\overline{\Delta \rho} / \rho_{0}=\left(\rho_{0}-\bar{\rho}\right) / \rho_{0}$ represents the mean normalized density reduction in the layer due to heat release, where $\vec{\rho}$ is the mean density in the layer and $\rho_{0}=$ $\frac{1}{2}\left(\rho_{1}+\rho_{2}\right)$ is the average (cold) density of the free streams. The mean density is defined here as

$$
\bar{\rho}=\rho_{0} \int_{\eta_{2}}^{\eta_{1}} \frac{T_{0}}{T_{0}+\overline{\Delta T}} d \eta
$$

where $\eta_{1,2}$ are the $1 \%$ points of the mean temperature profile on the high- and lowspeed sides, respectively (see figure 1), $T_{0}$ is the ambient temperature, and $\overline{\Delta T}$ is the time-averaged temperature rise at each point across the layer. This calculation neglects the small changes in pressure across the layer by taking the pressure to be constant. Small variations in the free-stream speed and density ratios from run to run (see Hermanson, Mungal \& Dimotakis 1987) have been corrected for by use of a formula proposed by Dimotakis (1986)

$$
\frac{\delta}{x}=\epsilon\left(\frac{1-r}{1+s^{\frac{1}{2}} r}\right)\left(1+s^{\frac{1}{2}}-\frac{1-s^{\frac{1}{2}}}{1+2.9 \frac{1+r}{1-r}}\right),
$$

where $s=\rho_{2} / \rho_{1}, r=U_{2} / U_{1}$, and $\epsilon$ is a constant. The correction consisted of using (4) to normalize each point in figure 3 by the ratio of the expected thickness for a cold layer with the actual speed and density ratio to that of a cold layer with the nominal values of $s=1, r=0.4$. A linear least-squares fit to the data in figure 3 suggests that the layer thinning, for a mean density reduction of $40 \%$, may be as high as $15 \%$. Extrapolation of the data in figure 3 indicates that the layer will retain a finite thickness even for the extreme case of zero mean density in the layer $\left(\overline{\Delta \rho} / \rho_{0}=1.0\right)$. The largest mean density reduction presented in this work, $\overline{\Delta \rho} / \rho_{0}=0.38$, corresponds to a run with an adiabatic flame temperature rise of $\Delta T_{\mathrm{f}}=940 \mathrm{~K}$ and a mean temperature rise in the layer of $\langle\overline{\Delta T}\rangle=248 \mathrm{~K}$. No dependence of the thinning trend on stoichiometric mixture ratio (at a given value of $\overline{\Delta \rho} / \rho_{0}$ ) was observed. 
The decrease in layer width with increasing heat release was noted by Wallace (1981) and was confirmed in the present set of experiments, in which the maximum mean flow temperature increase was about three times greater than that of Wallace. The implication of the results presented in figure 3 is that the rate at which the layer grows with downstream distance is reduced by heat release. The parameter $\delta_{1} /\left(x-x_{0}\right)$ represents the growth rate of a linearly growing shear layer. The location of the virtual origin, $x_{0}$, is a complicating factor, and the trend in layer growth rate suggested here does allow the possiblity that a shift in the virtual origin with heat release could result in slightly different growth rates than those suggested by figure 3. The location of the virtual origin in this work was determined visually from the intersection of the apparent layer edges, as revealed by spark schlieren photographs (see Brown \& Roshko 1974). This yielded values of $x_{0}$ in the range $-5<x_{0}<-1 \mathrm{~cm}$, but did not, however, suggest any systematic change in the location of the virtual origin with heat release. A representative value of $x_{0}=-3.2 \mathrm{~cm}$ was used for all normalizations in this investigation. It should be noted that initial conditions can have a significant effect on layer growth as has been shown, for example, by Batt (1975) and Browand \& Latigo (1979). In this investigation the initial conditions were essentially fixed.

Although the actual thickness of the layer decreased slightly with increasing heat release, the density decrease in the layer due to the increased temperature was nevertheless responsible for a displacement velocity in the external flow. At zero pressure gradient, this displacement deflected the low-speed free stream away from the layer and also deflected the layer away from the high-speed upper wall (the latter effect is a consequence of maintaining a fixed high-speed upper-wall orientation). The low-speed lower-wall divergence required for zero streamwise pressure gradient is, neglecting wall boundary layers, a direct measure of the displacement thickness of the layer, $\delta^{*}$, where $\delta^{*} /\left(x-x_{0}\right)$ indicates the tangent of the angle, $\beta$, by which the low-speed free streamline is deflected owing to the presence of the shear layer (see figure 1). Note that the displacement thickness is less than zero for a layer with no heat release, and increases steadily with heat release, as shown in figure 4 . Some unpublished data of Mungal are included in the figure with the present results. Since the streamwise pressure gradient for a given run was typically not exactly zero (see table $2, \S 8.1$ ), each data point in figure 4 was corrected empirically to correspond to the approximate wall setting that would have resulted in a zero value of pressure gradient.

The slight reduction in layer thickness (and growth rate) with increasing heat release is also confirmed by the mean velocity data. The shear-layer vorticity thickness, $\delta_{\omega}$, can be defined by normalization of the maximum slope of the mean velocity profile by the free-stream velocity difference, as follows:

$$
\frac{1}{\delta_{\omega}}=\frac{1}{\Delta U}\left(\frac{\mathrm{d} U}{\mathrm{~d} y}\right)_{\max } .
$$

A plot of the vorticity thickness variation with heat release, again corrected for small variations in speed ratio and density ratio, is shown in figure 5. The solid line represents the change in vorticity thickness, $\delta_{\omega}$, implied by the reduction in $1 \%$ thickness, $\delta_{1}$, assuming that $\delta_{\omega} / \delta_{1} \approx 0.55=$ constant. Brown \& Roshko (1974) report a somewhat lower value of $\delta_{\omega} / \delta_{1} \approx 0.48$. Each point was normalized by a representative value for the vorticity thickness at zero heat release. The portion of the present results at moderate heat release, including some unpublished data of 


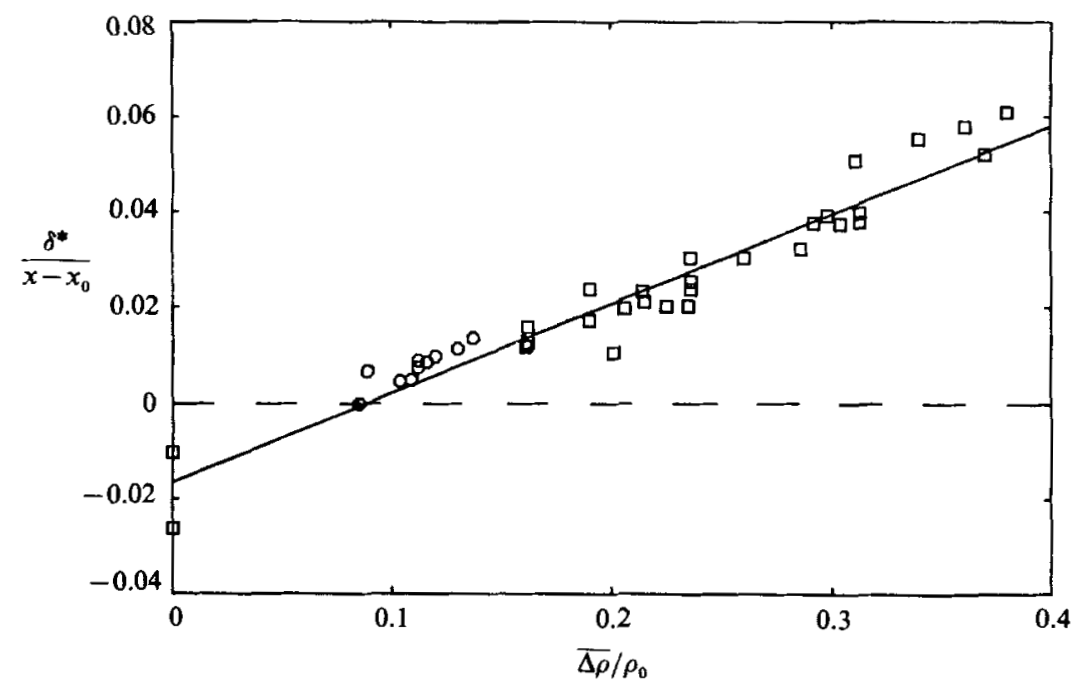

FIgURE 4. Normalized layer displacement vs. heat release: $\bigcirc$, Mungal (unpublished data);

$\square$, present results.

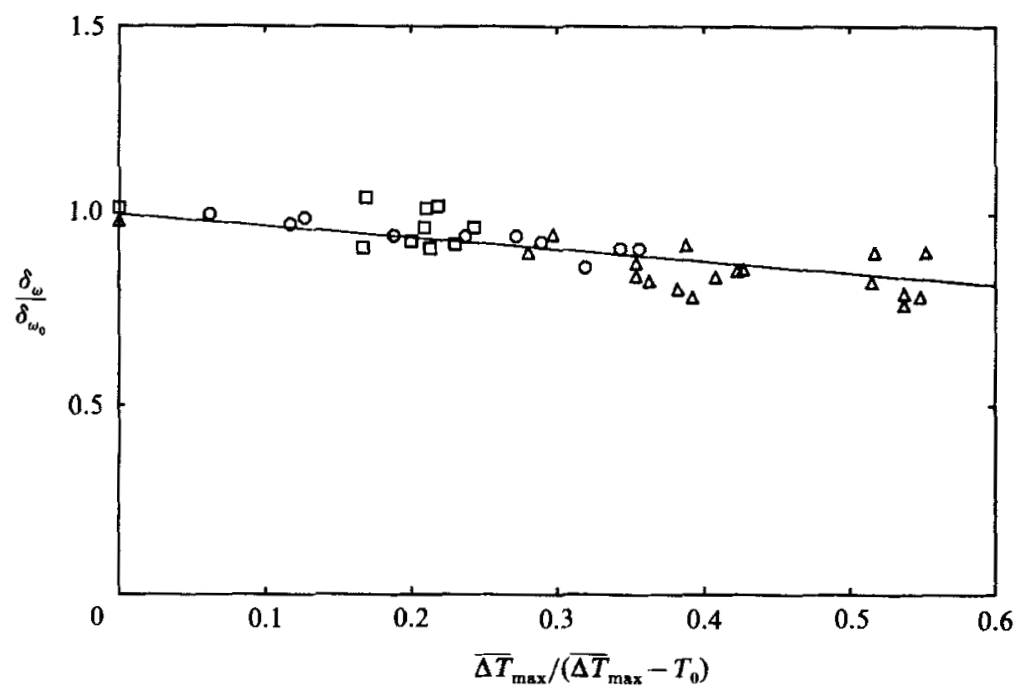

FIGURE 5. Normalized vorticity thickness vs. heat release: $\bigcirc$, Wallace (1981); $\square$, Mungal (unpublished data); $\Delta$, present results. $\delta_{\omega_{0}}$ is vorticity thickness at zero heat release.

Mungal, are in good agreement with Wallace. Since the data of Wallace were originally given in terms of $\overline{\Delta T}_{\max } / T_{0}$, the maximum time-averaged temperature rise over the ambient temperature, it was necessary to use the quantity $\overline{\Delta T}_{\max } /$ $\left(\overline{\Delta T}_{\max }+T_{0}\right)$ for the abscissa parameter of figure 5. This quantity is slightly different from $\overline{\Delta \rho} / \rho_{0}$ because the density is not a linear function of the temperature rise.

In addition to an increase in maximum slope with heat release, the mean velocity profile was also observed to change somewhat in shape. Velocity profiles at low and high heat release are plotted in figure 6 , against the normalized transverse coordinate $\left(y-y_{\mathrm{c}}\right) / \delta_{t y}$, where $y_{\mathrm{c}}$ is the location of the centre of each profile (i.e. where 


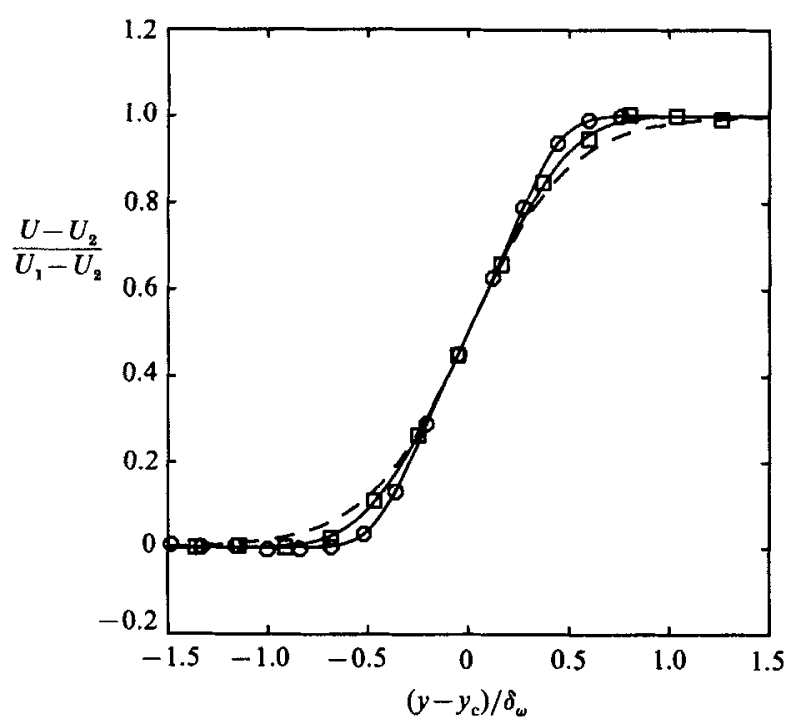

FIGURE 6. Velocity profile shape change with heat release : $\bigcirc$, unreacting flow, $\delta_{\omega} /\left(x-x_{0}\right)=0.090$; $\square$, reacting flow, $\Delta T_{\mathrm{f}}=457 \mathrm{~K}, \delta_{\omega} /\left(x-x_{0}\right)=0.079$. Dashed line is hyperbolic tangent profile.

$\left.U=\frac{1}{2}\left(U_{1}+U_{2}\right)\right)$. The dashed line represents a hyperbolic tangent profile with the same maximum slope as the velocity profiles. The profile at high temperature appears fuller than the corresponding low-temperature profile. A modified fit of the form

$$
\frac{U\left(y-y_{\mathrm{c}}\right)-U_{1}}{U_{1}-U_{2}}=\frac{1}{2}+\frac{1}{2} \tanh \left[2\left(\frac{y-y_{\mathrm{c}}}{\delta_{\omega}}\right)+\hat{b}\left(\frac{y-y_{\mathrm{c}}}{\delta_{\omega}}\right)^{3}\right]
$$

provides a good fit to mean velocity profiles over all temperatures in this investigation. The parameter $\hat{b}$ can be regarded as a measure of how the profile differs in fullness from a pure hyperbolic-tangent profile; $\hat{b}$ decreases with increasing temperature as the profile becomes more like a hyperbolic tangent.

Experiments performed at higher temperatures than those in this work by Pitz \& Daily (1983) in a combusting mixing layer formed downstream of a rearward-facing step indicated that the vorticity thickness did not appear to change between their cold runs and high-heat-release runs. Keller \& Daily (1983), however, report that in a reacting mixing layer between a cold premixed reactant stream and a preheated combustion product stream the vorticity thickness increased significantly with increasing temperature. Direct numerical simulations of two-dimensional flow in a reacting mixing layer performed by McMurtry et al. (1986) suggest a decrease in layer growth rate when exothermic reactions occur, in qualitative agreement with the present results. Similar trends have been seen in subsequent three-dimensional simulations by McMurtry \& Riley (1987). Additional discussion of heat release effects on layer growth is presented in $\$ 5.4$.

\subsection{Shear-layer entrainment}

That the layer growth rate does not increase with increasing temperature, in conjunction with a substantially reduced density in the layer, implies that the total volumetric entrainment of free-stream fluid into the layer must be reduced by heat release. A method which approximates the overall entrainment is to use the 


\begin{tabular}{lrrcccc}
\hline$\% \mathrm{~F}_{2} / \% \mathrm{H}_{2}$ & $\Delta T_{\mathrm{f}}(\mathrm{K})$ & $\overline{\Delta \rho} / \rho_{0}$ & $\eta_{1}$ & $\eta_{2}$ & $\beta$ & $r$ \\
& & & & & & \\
$1 / 1$ & 93.0 & 0.085 & 0.0734 & -0.0842 & 0.0103 & 0.388 \\
$1 / 8$ & 165.0 & 0.120 & 0.0717 & -0.0835 & 0.0174 & 0.380 \\
$2 / 2$ & 186.3 & 0.153 & 0.0632 & -0.0819 & 0.0221 & 0.411 \\
$2 / 4$ & 247.6 & 0.190 & 0.0642 & -0.0881 & 0.0179 & 0.373 \\
$3 / 3$ & 278.1 & 0.206 & 0.0615 & -0.0870 & 0.0216 & 0.392 \\
$4 / 4$ & 368.2 & 0.260 & $\mathbf{0 . 0 6 0 8}$ & -0.0897 & 0.0334 & 0.390 \\
$5 / 5$ & 456.8 & 0.286 & 0.0525 & -0.0945 & 0.0329 & 0.393 \\
$4 / 16$ & 600.3 & 0.311 & $\mathbf{0 . 0 5 4 4}$ & -0.0951 & 0.0509 & 0.373 \\
$6 / 6$ & 553.8 & 0.313 & 0.0520 & -0.0943 & 0.0436 & 0.382 \\
$6 / 12$ & 737.4 & 0.361 & $\mathbf{0 . 0 4 8 4}$ & -0.1001 & 0.0619 & 0.364
\end{tabular}

TABLE 1. Selected values of layer geometry parameters. $\Delta T_{\mathrm{f}} \equiv$ adiabatic flame temperature rise; $\overline{\Delta \rho} / \rho_{0} \equiv$ mean density reduction; $\eta_{1}, \eta_{2} \equiv$ similarity coordinate at $1 \%$ points of mean temperaturerise profiles on the high-speed and low-speed sides, respectively; $\beta \equiv$ low-speed lower sidewall deflection angle; $r \equiv$ free-stream speed ratio. The first two data points listed are from Mungal (unpublished data).

geometry of the layer as shown in figure 1 to derive (Brown 1978; Dimotakis 1986)

$$
\frac{\dot{V}}{U_{1}\left(x-x_{0}\right)}=\eta_{1}-r\left(\eta_{2}+\tan \beta\right),
$$

where $\dot{V}$ is the volume flux into the layer per unit span, $r=U_{2} / U_{1}, \eta_{1,2}$ are the similarity coordinate edges of the shear layer and $\beta$ is the deflection angle of the lowspeed lower wall. Certain assumptions about the nature of the vertical component of velocity are implicit in this formulation. Equation (5) neglects the displacement of the wall boundary layers by taking the free-stream vertical component of velocity to be zero at the high-speed upper wall and to have the value implied by the angle $\beta$ near the low-speed lower wall. These values of the free-stream vertical velocity are taken in (5) to also be those at the corresponding layer edges. By continuity, the change in mean horizontal velocity across the layer implies a corresponding change in the mean vertical velocity. At the outer edges of the layer, for example at the locations indicated by the $1 \%$ points in the mean temperature profiles, the mean vertical velocity components do have values nearly equal to those in the free stream. That is not the case, however, for points deeper inside the layer. Equation (5) will be referred to as the geometric entrainment calculation method. Representative values of $\eta_{1}, \eta_{2}$, and $\beta$ are presented in table 1 for varying amounts of heat release.

The entrainment into the layer can also be calculated from the mean velocity and density (i.e. temperature) profiles as follows:

$$
\frac{\dot{V}}{U_{1}\left(x-x_{0}\right)}=\int_{\eta_{2}}^{\eta_{1}} \frac{\rho U}{\rho_{0} U_{1}} \mathrm{~d} \eta .
$$

Unlike the geometric relation (5), the integral relation (6) does not make any assumptions about the nature of the vertical velocity components. This expression assumes that the layer is self-similar at the station at which the integral is performed. Results from Mungal et al. (1985) suggest that there is a weak Reynolds-number dependence on product formation. Since the growth rate does appear to be a function of the product formation (i.e. heat release), strictly speaking, the flow cannot be 
expected to be exactly self-similar. The quantity $\rho U$ was estimated by $\bar{\rho} U$, which was used here as an approximation for the density-velocity correlation $\overline{\rho u}$, where $u$ and $U$ are the instantaneous and mean downstream velocity components, respectively. Equation (6) will be referred to as the integral entrainment calculation method.

A common difficulty of both the integral and geometric entrainment calculation methods involves selection of appropriate values for $\eta_{1}$ and $\eta_{2}$. Konrad (1976) was able to circumvent the difficulty of edge point selection by incorporating an estimate for the intermittency obtained from species concentration data. The resulting expression for the entrainment can be expressed in integral form as

$$
\frac{\dot{V}}{U_{1}\left(x-x_{0}\right)}=\int_{-\infty}^{+\infty} \hat{\gamma}(\eta) \frac{\rho U}{\rho_{0} U_{1}} \mathrm{~d} \eta,
$$

where $\hat{\gamma}(\eta)$ is the intermittency, that is, the mean fraction of the time that turbulent (or mixed) fluid is encountered. Expressions (5) and (6) can be thought of as indicating the amount of fluid that is entrained into the overall boundaries of the layer; (7) estimates the amount that actually enters the turbulence (and becomes mixed). Equation (7) will be referred to as the intermittency-weighted entrainment calculation method.

An estimate for the intermittency can be made by relating it to the probability of finding mixed fluid at a given location in the layer. Koochesfahani \& Dimotakis (1986) showed that the total mixed-fluid probability, $P_{\mathrm{m}}(y)$, can be related to the sum of the normalized (mean) product concentrations, $\overline{C_{\mathrm{p}}}$, at very high and very low stoichiometric mixture ratios, as follows:

$$
P_{\mathrm{m}}(y)=\frac{\overline{c_{\mathrm{P}}}(y ; \phi=\infty)}{c_{01}}+\frac{\overline{c_{\mathrm{P}}}(y ; \phi=0)}{c_{02}} .
$$

The mean product concentration can, at low heat release, be related to the mean temperature rise (see Mungal \& Dimotakis 1984), giving for the mixed-fluid probability

$$
P_{\mathrm{m}}(y)=\frac{\hat{C}_{p}}{\Delta Q} \frac{\overline{\Delta T}(y ; \phi=\infty)}{c_{01}}+\frac{\hat{C}_{p}}{\Delta Q} \frac{\overline{\Delta T}(y ; \phi=0)}{c_{02}},
$$

where $\Delta Q$ is the molar heat release of the chemical reaction and $\hat{C}_{p}$ is the molar specific heat capacity. It was shown by Mungal \& Dimotakis (1984) that the amount of product formed is close to the asymptotic limits for $\phi=8$ and $\phi=\frac{1}{8}$. These limits mean physically that the (mixed) lean reactant has been nearly fully consumed. Taking, as an approximation, the intermittency to be equal to the total mixed-fluid probability gives, in similarity coordinates,

$$
\hat{\gamma}(\eta ; \hat{q}) \approx \frac{\hat{C}_{p}}{\Delta Q} \frac{\overline{\Delta T}(\eta ; \phi=8)}{c_{01}}+\frac{\hat{C}_{p}}{\Delta Q} \frac{\overline{\Delta T}\left(\eta ; \phi=\frac{1}{8}\right)}{c_{02}} .
$$

The parameter $\hat{q}$ is added to indicate a possible functional dependence on heat release; a possible choice is $\hat{q} \equiv \overline{\Delta \rho} / \rho_{0}$.

It is not clear exactly how intermittency changes with heat release, as in this investigation the cases $\phi=8$ and $\phi=\frac{1}{8}$ were not investigated at high heat release. It will be seen in $\S 6$, however, that there is some decrease in the heights of the mean temperature-rise profiles normalized by the adiabatic flame temperature rise, with heat release. This allows the possibility that intermittency may be affected by heat release. 


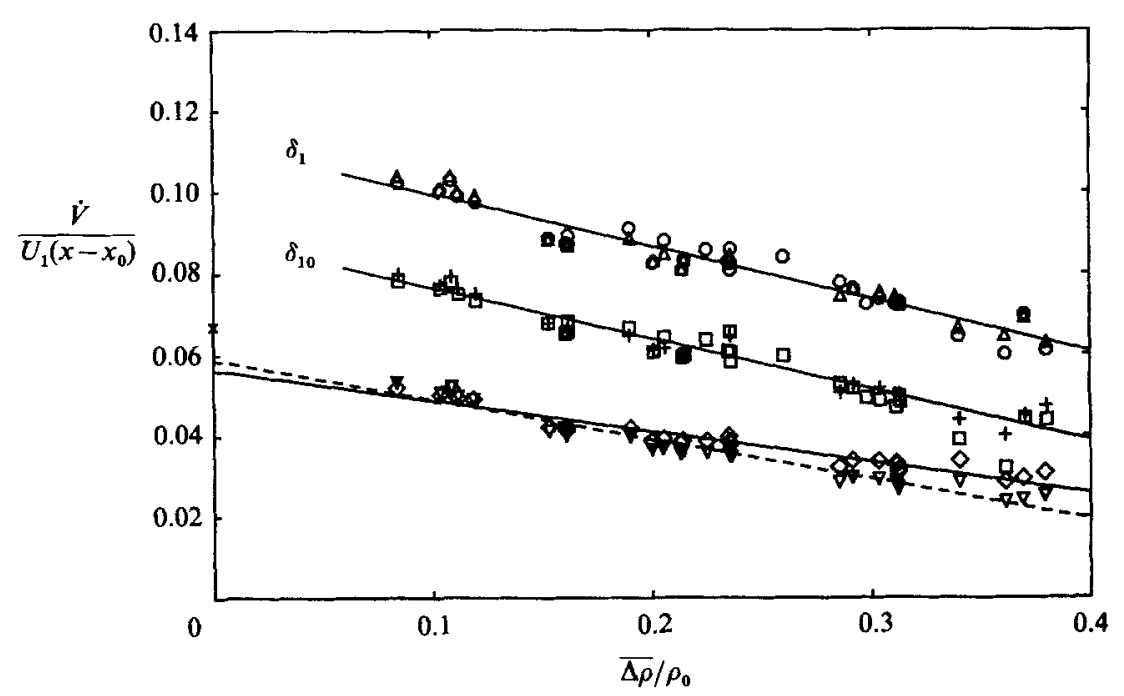

Figure 7. Dependence of volumetric entrainment on heat release : $\bigcirc, \square$, geometric entrainment calculation method; $\triangle,+$, integral entrainment calculation method; $-\diamond-$, intermittencyweighted method using $\hat{\gamma}(y: 0) ;-\nabla-$, intermittency-weighted method using $\hat{\gamma}(\boldsymbol{y} ; \hat{q}) ; \times, \mathrm{Konrad}$ (1976). See text for explanation. Data for $0<\overline{\Delta \rho} / \rho_{0}<0.13$ calculated from Mungal (unpublished data).

The resulting entrainment data, using the geometric, integral, and intermittencyweighted entrainment calculation methods, are presented in figure 7 . Results calculated from unpublished data of Mungal are also included. The data sets labelled $\delta_{1}$ and $\delta_{10}$ were calculated without intermittency using for edge points the $1 \%$ and $10 \%$ points in the mean temperature profiles, respectively. The solid and dashed lines are linear least-squares fits to the data.

The lowest sets of data in figure 7 were calculated by the intermittency-weighted method. The intermittency used in calculating the data set labelled $\hat{\gamma}(y ; 0)$ was estimated using the low-heat-release results of Mungal \& Dimotakis and (10). For the data set labelled $\hat{\gamma}(y ; \hat{q})$, the height of the intermittency profile was taken to decrease in direct proportion to the decrease in the normalized mean temperature profiles at $\phi=\frac{1}{4}$ with heat release, as discussed in $\S 6$. The data with changing intermittency do not suggest a significantly different trend than the results calculated incorporating the intermittency estimated from the low-heat-release results. It should be emphasized that this change in intermittency with heat release is speculative and that the actual dependence of the intermittency on temperature is yet to be established.

Regardless of the choice of method or reference points, the inference is that the total entrainment into the layer is strongly reduced with increasing heat release, amounting to a decrease of about $50 \%$, for a mean density in the layer of $40 \%$ below its nominal cold value. That the entrainment reduction is in excess of the mean density reduction suggests that the decrease in entrainment flux with heat release more than compensates for the displacement effects due to density change, yielding a thinner layer thereby. The entrainment reported by Konrad (1976) for a nonreacting shear layer, estimated using intermittency, is included for comparison in figure 7. The value shown was scaled for comparison by the estimated growth rate at zero heat release in the present experiment, and is in fair agreement with the 
present results. The linear least-squares fits shown in figure 7 , if extrapolated to higher values of mean density reduction, would cross the $x$-axis and indicate zero entrainment into the layer for a value of $\overline{\Delta \rho} / \rho_{0} \approx 0.8$. It will be argued in $\S 7$ that $\overline{\Delta \rho} / \rho_{0}$ may never reach that value, even for very high flame temperatures but will rather tend to a limiting value that is substantially less.

The difference between the integral and geometric methods can be used to infer, in an approximate fashion, the amount by which the time-averaged quantity $\overline{\rho u}$ differs from the approximation $\bar{\rho} U$ used for the computations in this section. The difference is the fluctuation correlation term $\overline{\rho^{\prime} u^{\prime}}$. In view of the good agreement between the integral and geometric methods shown in figure 7 over all values of heat release, the quantity $\bar{\rho}^{\prime} u^{\prime} / \bar{\rho} U$ does not appear to be greater than about $4 \%$ over the width of the layer. If it is assumed that the profile $\overline{\rho^{\prime} u^{\prime}}(\eta)$ is Gaussian-like, the maximum error near the centerline of the layer could be about $9 \%$. Since the mean velocity itself is estimated to be accurate only to about $4 \%$ (Rebollo 1973) however, the data do allow the possibility that $\overline{\rho^{\prime} u^{\prime}}$ could on average be much smaller than $4 \%$ of $\rho U$, or, in fact, zero.

\subsection{Entrainment ratio}

Methods analogous to the integral and geometric methods for total entrainment can be developed to give estimates of the volumetric entrainment ratio, that is, the ratio of high-speed entrained fluid to low-speed entrained fluid. The geometric result is developed in Brown (1978) and Dimotakis (1986) and is

$$
E_{v} \approx \frac{U_{1}}{U_{2}} \frac{\eta_{1}}{-\eta_{2}-\tan \beta}
$$

As noted by Dimotakis, (11) assumes that the ratio of the fluid inducted into the layer from each of the free streams is the same as the ratio of the fluid fluxes intercepting the corresponding (mean) visual edges of the shear layer. For the entrainment calculations in this investigation, the edges of the layer were taken to be those indicated by specific points in the mean temperature profiles, as explained earlier. An integral method for estimating the entrainment velocities into the layer can be derived from formulas presented in Konrad (1976), here expressed in terms of linear similarity coordinates:

and

$$
\frac{V_{\mathrm{e}_{1}}}{U_{1}}=\int_{\eta_{0}}^{\eta_{1}} \frac{\rho U}{\rho_{0} U_{1}} \mathrm{~d} \eta
$$

where $V_{\mathrm{e}_{1.2}}$ are the entrainment velocities into the high-speed and low-speed sides of the layer, respectively, and $\eta_{0}$ is the location of the dividing streamline. $\eta_{0}$ is obtainable from the following commonly employed relation, derived from combining the continuity and momentum equations;

$$
\int_{-\infty}^{\eta_{0}} \frac{\rho U}{\rho_{0} U_{1}}\left(\frac{U}{U_{1}}-r\right) \mathrm{d} \eta=\int_{\eta_{0}}^{\infty} \frac{\rho U}{\rho_{0} U_{1}}\left(1-\frac{U}{U_{1}}\right) \mathrm{d} \eta
$$

The corresponding integral formulas incorporating intermittency are

$$
\frac{V_{e_{1}}}{U_{1}}=\int_{\eta_{0}}^{\infty} \hat{\gamma}(\eta) \frac{\rho U}{\rho_{0} U_{1}} \mathrm{~d} \eta
$$




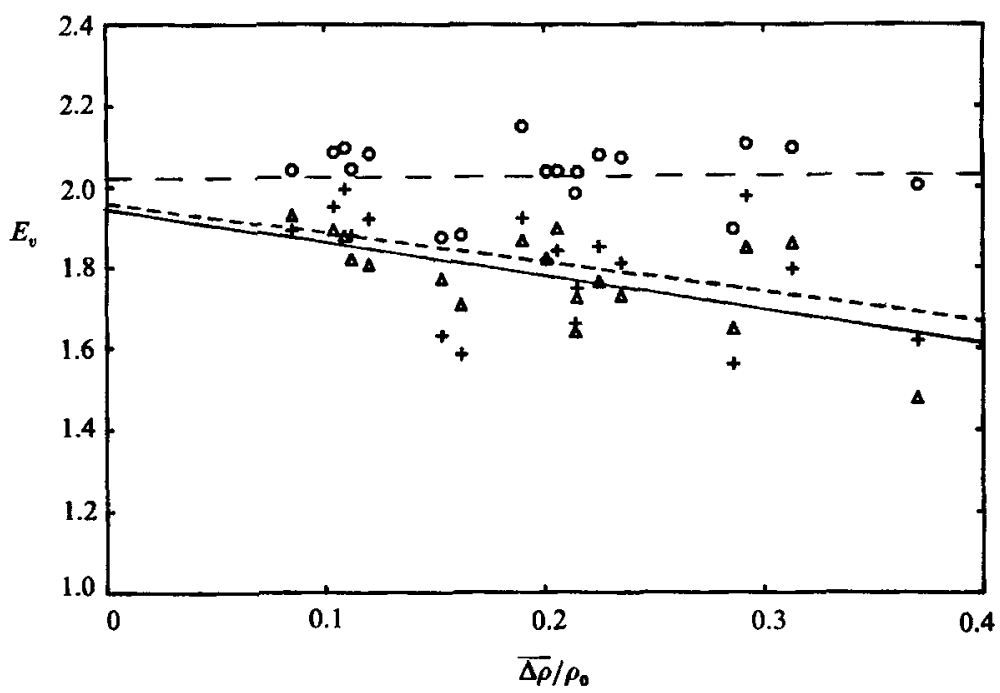

Figure 8. Effect of heat release on entrainment ratio: $O$, based on $1 \%$ thickness of mean temperature profiles; $\triangle$, based on $10 \%$ thickness; + , calculated with intermittency. Lines shown are least-squares fit to data.

and

$$
\frac{V_{\mathrm{e}_{2}}}{U_{1}}=\int_{-\infty}^{\eta_{0}} \hat{\gamma}(\eta) \frac{\rho U}{\rho_{0} U_{1}} \mathrm{~d} \eta .
$$

The volumetric entrainment ratio is given by

$$
E_{v}=\frac{V_{\mathrm{e}_{1}}}{V_{\mathrm{e}_{\mathrm{z}}}}
$$

The issue again arises, for the calculations without intermittency, of how to choose the values of $\eta_{1}$ and $\eta_{2}$. This issue is much more critical here than in the evaluation of the methods for the total entrainment, since small inaccuracies in $V_{\mathrm{e}_{1}}$ and $V_{\mathrm{e}_{2}}$ (resulting from errors in $\eta_{1}$ and $\eta_{0}$, for example) can result in large errors in the ratio $E_{v}$. The fact that only eight temperature probes were employed across the width of the layer made precise determination of the values of $\eta_{1}$ and, to a lesser extent, $\eta_{10}$ difficult in this investigation. Note that the same cautionary remarks made in the previous section concerning the nature of the mean vertical velocity components also apply to the present discussion. In particular, in the geometric calculation (11) the boundary layer on the low-speed lower wall adds to the apparent layer displacement and can result in an observed value of $\beta$ which exceeds the actual $\delta^{*} /\left(x-x_{0}\right)$ of the layer. This could cause (11) to indicate a substantially higher entrainment ratio than actually occurs in the flow.

The calculations from the present data and also those of Mungal using integral entrainment formulas, (12) and (13), are presented in figure 8, using as before the $1 \%$ and $10 \%$ points of the mean temperature profile to get $\eta_{1}$ and $\eta_{2}$. Linear least-square fits were performed for each data set. Each data point in figure 8 has been corrected for run-to-run variations in speed and density ratio by using, in a similar fashion to the growth rate correction applied in $\$ 5.1$, the following formula derived by Dimotakis (1986) for the entrainment ratio:

$$
E_{v} \approx s^{\frac{1}{4}}\left(1+0.68 \frac{1-r}{1+r}\right)
$$


The $1 \%$ data indicate no systematic change in volumetric entrainment ratio. The corresponding $10 \%$ results indicate a decrease in volumetric entrainment ratio with heat release of up to about $15 \%$ for a mean density reduction in the layer of $40 \%$. The results employing the integral formulas with intermittency, (15) and (16), are also shown in figure 8 and indicate a comparable trend with the results calculated using the $10 \%$ points of the mean temperature profile. In this calculation, the intermittency was taken to be unchanging with heat release. An additional estimate of the change in entrainment ratio with heat release, using large-scale structure statistical information, is presented in $\$ 7$, and is consistent with these results.

It is worth noting that the actual values of the entrainment ratio estimated by the methods without intermittency, using the $1 \%$ points, appear to exceed substantially the values calculated taking intermittency into account, and also the value given by Konrad of $E_{v} \approx 1.3$ for the non-reacting layer at comparable speed ratio. This suggests that the amount of high-speed fluid within the outside boundaries of the layer (as suggested by the width of the mean temperature profile), but yet unmixed, may exceed the corresponding amount of low-speed fluid by a factor greater than 1.3 .

\subsection{Discussion of heat release effects on layer growth}

The observed heat release effects on layer growth and entrainment may be related to a decrease in the turbulent shear stress in the layer. The experimental data in fact suggest a decrease in shear stress which can be attributed to the density reduction in the layer caused by heat release. The following arguments are included as an $a$ posteriori effort to estimate the magnitudes of the various effects that enter in the accounting of the growth rate of the shear layer. The discussion describes what is essentially a self-consistent calculation; it is not proposed here that such arguments could have been used to predict these results prior to their establishment by experiment.

The turbulent shear stress can be calculated from the time-averaged equations for conservation of mass and $x$-momentum. These equations for this flow can be developed by expressing the components of velocity and density as the sum of mean and fluctuating parts, i.e. $\tilde{u}=U+u^{\prime}, \tilde{v}=V+v^{\prime}$, and $\tilde{\rho}=\rho+\rho^{\prime}$, where the primes indicate the fluctuating parts. The mean quantities are shown here with no diacritical marks. The equations are

$$
\begin{gathered}
\frac{\partial}{\partial x}(\rho U)+\frac{\partial}{\partial y}(\rho \tilde{V}) \approx 0, \\
\frac{\partial}{\partial x}\left(\rho U^{2}\right)+\frac{\partial}{\partial y}(\rho U \tilde{V}) \approx-\frac{\partial p}{\partial x}+\frac{\partial \tau}{\partial y},
\end{gathered}
$$

where $\rho \tilde{V}=\rho V+\overrightarrow{\rho^{\prime} v}$, the bar being used to denote the time average. It is assumed here (as supported by the discussion in $\$ 5.2$ ) that $\overline{\rho^{\prime} u^{\prime}} \ll \rho U$, that the Reynolds stresses are much larger than the viscous stresses, and that all fluctuation correlations in $u\left(\rho \overline{u^{\prime 2}}, 2 U \overline{\rho^{\prime} u^{\prime}}\right.$, and $\left.\overline{\rho^{\prime} u^{\prime 2}}\right)$ are small compared with the product of $\rho U^{2}$. The gradients in the $y$-direction are assumed to be much larger than gradients of similar quantities in the $x$-direction. The quantity $\tau$ in the $x$-momentum equation (19) is the turbulent shear stress, where $\tau=-\rho \overline{u^{\prime} v^{\prime}}$, the Reynolds stress, plus higher-order terms $\left(\overline{\rho^{\prime} u^{\prime} v^{\prime}}, U \overline{\rho^{\prime} v^{\prime}}\right.$, and $\left.V \overline{\rho^{\prime} u^{\prime}}\right)$. For the purposes of this discussion, it is not necessary to state exactly which fluctuation terms constitute $\tau$.

The $x$-momentum and continuity equations can be combined by introducing a 
similarity variable and also a stream function. Taking here $\eta \equiv y / \delta(x) \dagger$, where $\delta(x)$ is a characteristic layer width (e.g. $\delta_{1}$ ), allows rewriting of the $x$-momentum equation (19) to give

$$
-\eta \rho U \frac{\mathrm{d} U}{\mathrm{~d} \eta} \frac{\mathrm{d} \delta}{\mathrm{d} x}+\rho \widetilde{V} \frac{\mathrm{d} U}{\mathrm{~d} \eta}=\frac{\mathrm{d} \tau}{\mathrm{d} \eta}-\eta \frac{\mathrm{d} p}{\mathrm{~d} \eta} \frac{\mathrm{d} \delta}{\mathrm{d} x} .
$$

The continuity equation (18) is identically satisfied if the two-dimensional mean velocity components are derived in the usual manner in terms of a stream function of the form $\Psi=\rho_{0} U_{1} \delta(x) f(\eta)$ where the velocity-density products are given by

$$
\rho U=\frac{\partial \Psi}{\partial y}, \quad \rho \tilde{V}=-\frac{\partial \Psi}{\partial x} .
$$

Expressing (21) in terms of linear similarity coordinates gives the following relations for the velocity components:

and

$$
U=U_{1} \hat{s} \frac{\mathrm{d} f}{\mathrm{~d} \eta}
$$

where $\hat{s}(\eta) \equiv \rho_{0} / \rho$. Combining (20) and (21) with (22) and (23) thus gives

$$
-\frac{U_{1}^{2}}{\Delta U^{2}} \frac{\mathrm{d} \delta}{\mathrm{d} x} f\left[\frac{\mathrm{d} \hat{s}}{\mathrm{~d} \eta} f+\hat{s} \frac{\mathrm{d}^{2} f}{\mathrm{~d} \eta^{2}}\right]=\frac{1}{\rho_{0} \Delta U^{2}} \frac{\mathrm{d} \tau}{\mathrm{d} \eta}-\frac{\eta}{\rho_{0} \Delta U^{2}} \frac{\mathrm{d} p}{\mathrm{~d} \eta} \frac{\mathrm{d} \delta}{\mathrm{d} x}
$$

Equation (24) provides, for given $f(\eta)$ and $\hat{s}(\eta)$ profiles, a means of estimating the shear-stress profile. The second term on the right-hand side (pressure term) is small.

Equation (24) can be integrated with respect to $\eta$ to determine the layer shearstress profile. Performing this calculation may result in a computed shear-stress profile that indicates a non-zero shear stress in one of the free streams (see, for example, Spencer \& Jones 1971). The calculated shear stress can be ensured to tend to zero in both free streams if the following condition, which is essentially a statement of conservation of mass for the top (i.e. high-speed) half of the layer, is enforced:

$$
\eta_{\mathrm{n}}=\int_{\eta_{0}}^{\eta_{\mathrm{h}}} \frac{\rho U}{\rho_{1} U_{1}} \mathrm{~d} \eta
$$

where $\eta_{\mathrm{h}}$ is an arbitrary point in the high-speed free stream outside the layer. The dividing streamline, $\eta_{0}$, corresponds to the location at which $\rho \tilde{V}=\eta \rho U$, and is also the point at which the shear stress in the layer is a maximum. In the computations of Lang (1985) for a non-reacting layer, the shear stress was fixed at zero in the free streams by specifying the value of the vertical velocity component in the lowspeed free stream. The vertical velocity component in the high-speed free stream was then obtained from integrating the continuity equation (18).

Calculated turbulent shear-stress profiles are shown in figure 9. Model density and velocity profiles, obtained from experimental results, provided the functions $f(\eta)$ and $\hat{s}(\eta)$. The changes in mean velocity profile with heat release were discussed in $\$ 5.1$; the changes in mean temperature profile will be discussed in $\$ 6$. For the purposes of

$\dagger$ In this discussion, this form is more convenient than the form $\eta=y /\left(x-x_{0}\right)$ used in previous sections. 


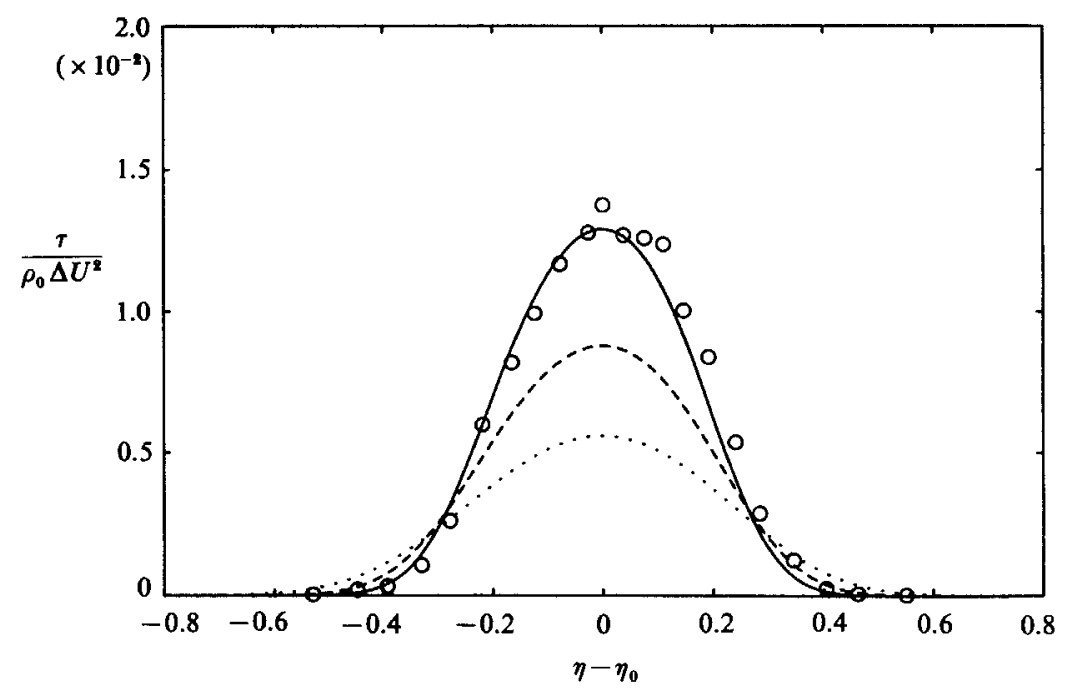

FigURE 9. Calculated shear-stress profiles:-, $\overline{\Delta \rho} / \rho_{0}=0 ; \cdots, \Delta \rho / \rho_{0}=0.2 ; \cdots, \overline{\Delta \rho} / \rho_{0}=0.4 ; \bigcirc$, data of Spencer \& Jones (1971). $\eta_{0}$ is the location of the dividing streamline.

the computations performed in this section, symmetric model density (i.e. temperature) and velocity profiles were assumed, whose fit parameters were varied to match the observed changes in the profiles with heat release. The growth rate, $\mathrm{d} \delta / \mathrm{d} x$, was taken from the data of $\delta_{1} /\left(x-x_{0}\right)$ depicted in figure 3 . It can be seen that with increasing heat release a marked decrease in shear stress is indicated. A broadening of the shear-stress profile with heat release is also apparent; this results from the broadening of the mean velocity profile $U(\eta)$ as was seen in figure 6 . The calculated shear stress at zero heat release agrees well with the values of $\tau=-\rho \overline{u^{\prime} v^{\prime}}$ reported by Spencer \& Jones (1971) for non-reacting flow (scaled here in width to match the present data, which are at a different speed ratio). A decrease in shear stress with heat release is also consistent with the results of direct numerical simulations by McMurtry \& Riley (1987), which indicate that a density reduction due to heat release serves to both suppress the turbulent shear stress as well as to lower the generation rate of turbulent kinetic energy.

It is interesting to note that the decrease in shear stress with heat release is also consistent with the mixing-length scaling argument of Prandtl (1925):

$$
\tau \approx \mu_{t} \frac{\partial U}{\partial y}, \quad \mu_{t}=\rho \hat{l}^{2}\left|\frac{\partial U}{\partial y}\right|
$$

where $\hat{l}$ is the mixing length. Expressing $\partial U / \partial y$ in similarity coordinates gives

$$
\tau \approx \rho \frac{\hat{l}^{2}}{\delta^{2}}\left|\frac{\mathrm{d} U}{\mathrm{~d} \eta}\right| \frac{\mathrm{d} U}{\mathrm{~d} \eta}
$$

Assuming $\hat{l} \sim \delta$, (26) suggests that if the similarity mean velocity profile is not strongly altered by heat release, the shear stress will decrease with decreasing density. An alternative argument is that if the correlation $\overline{u^{\prime} v^{\prime}}$ is not greatly altered by heat release, then the Reynolds stress, $\tau=-\rho \overline{u^{\prime} v^{\prime}}$, will decrease with heat release owing to the decreasing density. It should be pointed out, however, that some change in the velocity field does evidently occur with increasing heat release. This was seen 
in the present work by the broadening of the mean velocity profiles in figure 6 . An argument presented by Wallace (1981) suggests that the vorticity distribution within the large structures is altered by heat release owing to the action of the baroclinic torque which arises from non-aligned density gradient and acceleration vectors. This implies a change both in the mean velocity and in the fluctuating velocity correlation terms. The effects of thermal expansion in addition to those of baroclinic torque have been examined in detail by the direct numerical simulations of McMurtry et al. (1986) and McMurtry \& Riley (1987). The results of those simulations clearly show a redistribution of vorticity leading to a decrease in the vorticity in the structure cores. Some changes in the shape of the mean velocity profiles with heat release were also indicated by those simulations. However, as quantified in the following discussion, the density change alone appears to be sufficient to result in decreased values of turbulent shear stress consistent with the observed decrease in layer growth rate. This, in addition to the observed relative insensitivity of the mean velocity profile to heat release, suggests that, as concerns the shear stress, the change in density dominates over changes in the velocity field.

There appear to be several possible values of density to employ in estimating the turbulent shear-stress reduction caused by heat release. One choice is the mean density at the given transverse station in the layer. Another possibility is suggested by considering the large-scale nature of the flow. Rajagopalan \& Antonia (1981) found in a non-reacting flow that at least $80 \%$ of the total shear stress appeared to be contained within the large-scale structures. As discussed in $\$ 7$, large-scale structures persisted at all levels of heat release in this investigation. This suggests that an appropriate method for estimating the shear stress in a flow with heat release might consist of a combination of the shear stress found in unreacting flow and some representative large-scale structure shear stress. The shear stress could be expressed as

$$
\tau(\eta)=(1-\hat{a}) \tau_{0}(\eta)+\hat{a} \tau_{0}(\eta) \frac{\rho^{*}}{\rho_{0}}
$$

where $\tau_{0}(\eta)$ is the shear-stress profile for the non-reacting flow, $\rho^{*}$ is the structure density (here taken to be uniform within the structure), and $\hat{a}$ is the fraction of total stress contributed by the large-scale structure.

In order to obtain an estimate for the temperature in the structures from the mean temperature profile, it must be kept in mind, as pointed out by Mungal \& Dimotakis (1984), that cold tongues of fluid penetrate well into the layer. This will be seen in $\$ 6$ to also be the case at high heat release. These statements imply a value of a representative homogeneous large-scale structure temperature somewhat higher than the maximum mean temperature. The structure density can thus be expressed as

$$
\frac{\rho^{*}}{\rho_{0}}=\frac{T_{0}}{T_{0}+\hat{c} \overline{\Delta T}_{\max }}
$$

where $\overline{\Delta T}_{\max }$ is the maximum mean temperature rise in the layer and $\hat{c}$ is a weighting factor of value greater than unity.

The dependence of the peak shear stress on heat release is shown in figure 10 . The solid line represents the peak shear stress calculated from the mean velocity and density profiles. The dashed and dotted lines correspond to the peak shear stress predicted by the method of (27), assuming values of $\hat{a}=0.8, \hat{c}=1.0$ and $\hat{a}=1.0$, $\hat{c}=1.1$, respectively. These two curves are above and below the result calculated from the mean velocity and density profiles. This method does not allow deter- 


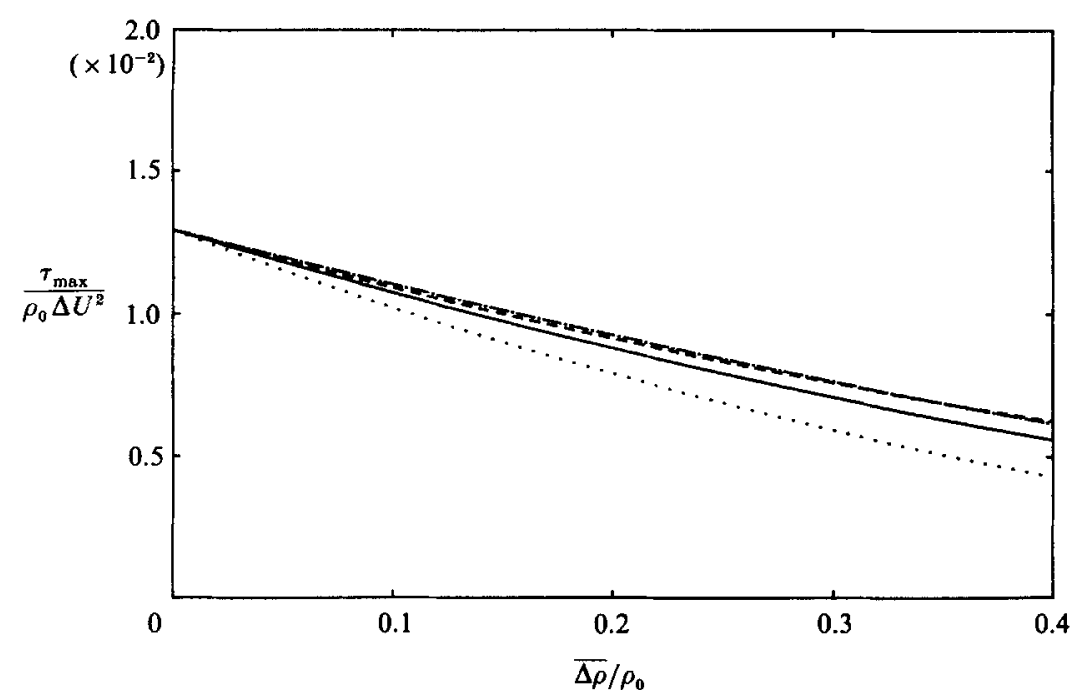

FIGURE 10. Reduction in maximum shear stress with heat release :-, calculated from $\rho, U$ profiles: $\cdots, \hat{a}=0.8, \hat{c}=1.0 ; \cdots, \hat{a}=1.0, \hat{c}=1.1 ;-.-, \delta / x=$ constant. See text for explanation.

mination of $\hat{a}$ and $\hat{c}$ separately. It does, however, suggest that the scaling of (27) is reasonable and is consistent with the possibility that at least $80 \%$ of the shear stress is contributed by the large-scale structures. Also included in figure 10 is the trend in maximum shear stress that would occur if the layer growth rate were constant and unchanged by heat release. The implication is that, even if the layer growth rate were constant, a substantial decrease in the turbulent shear stress would occur with heat release.

\section{Temperature rise and amount of product formation}

Time-resolved temperature measurements were performed using the cold-wire probes described in $\$ 2.2$. Sample time traces are presented in figure $11(a)$, corresponding to a run with $2 \% \mathrm{~F}_{2}$ and $2 \% \mathrm{H}_{2}$, with an adiabatic flame temperature rise of $186 \mathrm{~K}$. Similar traces were presented, for conditions of low heat release, by Mungal \& Dimotakis (1984). Figure $11(a)$ consists of individual traces representing the temperature rise recorded by the individual probes, with the high-speed free stream toward the top of the figure. Each horizontal axis represents $51.2 \mathrm{~ms}$ of real time. The vertical coordinate of each trace is $\Delta T / \Delta T_{\max }$, where $\Delta T_{\max }$ is the maximum temperature rise recorded by any probe. The traces are representations of the temperatures sensed by the probes as the hot and cold fluid elements flow past them; hence, the leading edge of any fluid element appears in the figure to the left of the corresponding trailing edge. Each time axis corresponds to a geometric distance which represents the product of the local flow velocity and the given time interval; to represent these distances each time axis would need to be shown substantially larger in the figure to be to scale with the probe spacings shown. The amount of compression in this sense ranges from a factor of about 9 for probes near the high-speed free-stream to roughly 4 for probes near the low-speed free stream. Time traces corresponding to a $4 \% \mathrm{~F}_{2}$ and $4 \% \mathrm{H}_{2}$ run $\left(\Delta T_{\mathrm{f}}=368 \mathrm{~K}\right)$ and a $6 \% \mathrm{~F}_{2}$ and $6 \% \mathrm{H}_{2}$ run $\left(\Delta T_{\mathrm{f}}=554 \mathrm{~K}\right)$ are shown in figures $11(b)$ and $11(c)$, respectively.

It can be observed in figure $11(a-c)$ that there are large, hot structures, separated 
(a)

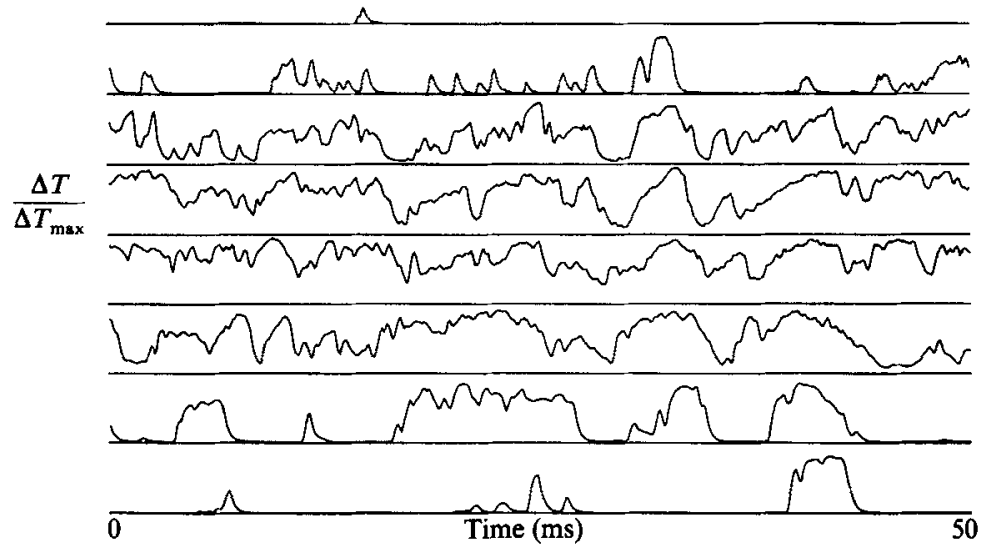

(b)
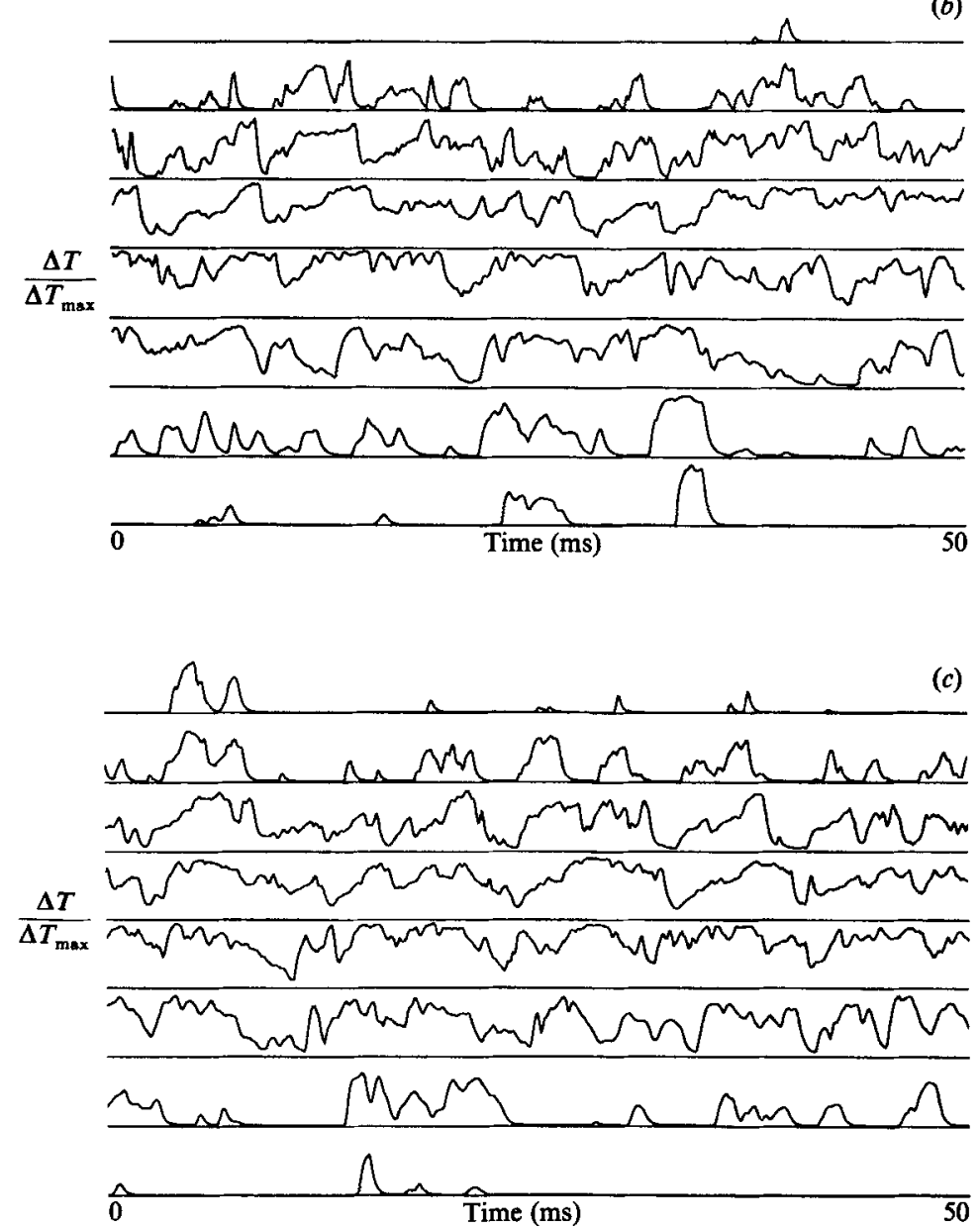

Figure 11. Temperature vs. time trace; $\phi=1$; high-speed fluid on top. $(a) 2 \% \mathrm{~F}_{2}: 2 \% \mathrm{H}_{2}$, $\Delta T_{\mathrm{f}}=186 \mathrm{~K}, \Delta T_{\max }=171 \mathrm{~K} .(b) 4 \% \mathrm{~F}_{2}: 4 \% \mathrm{H}_{2}, \Delta T_{\mathrm{f}}=368 \mathrm{~K}, \Delta T_{\max }=348 \mathrm{~K}$. (c) $6 \% \mathrm{~F}_{2}: 6 \% \mathrm{H}_{2}$, $\Delta T_{\mathrm{f}}=554 \mathrm{~K}$, probe $\Delta T_{\max }=496 \mathrm{~K}$. 


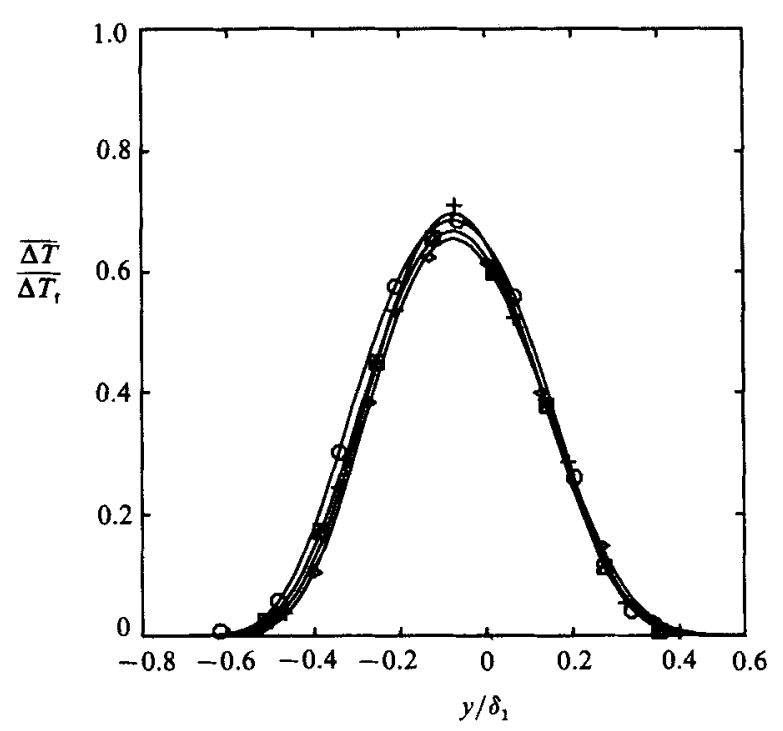

Fiaure 12. Mean temperature-rise profiles, $\phi=1: \bigcirc, 2 \% \mathrm{~F}_{2}: 2 \% \mathrm{H}_{2}, \overline{\Delta \rho} / \rho_{0}=0.162 ; \square, 3 \% \mathrm{~F}_{2}$ : $3 \% \mathrm{H}_{2}, \overline{\Delta \rho} / \rho_{0}=0.206 ; \Delta, 4 \% \mathrm{~F}_{2}: 4 \% \mathrm{H}_{2}, \overline{\Delta \rho} / \rho_{0}=0.260 ;+, 5 \% \mathrm{~F}_{2}: 5 \% \mathrm{H}_{2}, \Delta \rho / \rho=0.286 ; \diamond$, $6 \% \mathrm{~F}_{2}: 6 \% \mathrm{H}_{2}, \overline{\Delta \rho} / \rho_{0}=0.313$. Profiles shifted to coincide with peak of $2 \% \mathrm{~F}_{2}: 2 \% \mathrm{H}_{2}$ case.

by cold tongues of cool free-stream fluid which penetrate deep into the layer. These observations are consistent with the findings of Mungal \& Dimotakis (1984). The dynamics of the layer structures will be discussed in $\$ 7$. The convection velocity and mean structure spacing suggest that roughly seven large structures passed the measuring station during the time interval represented by each time trace in those figures. It should be noted that conduction error (Scadron \& Warshawsky 1952; Paranthoen, Lecordier \& Petit 1982) prevented the probes near the centre of the layer from indicating a zero temperature rise, which would correspond to the cool, unreacted tongues of recently entrained fluid.

The average, over an entire run, of all time trace records results in the mean temperature profile. Several profiles, normalized by the corresponding adiabatic flame temperatures, are shown in figures 12,13 and 14. Each of the figures presents profiles at a given stoichiometric mixture ratio $\left(\phi=1, \frac{1}{2}, \frac{1}{4}\right)$. The transverse coordinate, $y / \delta_{1}$, indicates the distances from the splitter-plate tip normalized by the corresponding value of $\delta_{1}$ for each run, with positive values toward the high-speed side of the layer. This coordinate was employed to enable easy comparison between profiles with different layer thicknesses. Following Mungal \& Dimotakis (1984), an empirical function of the form

$$
\frac{\Delta T}{\Delta T_{\mathrm{f}}}=\exp \left(c_{1}+c_{2} y+c_{3} y^{2}+c_{4} y^{3}+c_{5} y^{4}\right)
$$

was employed to represent the local mean temperature rise profile. The coefficients for the present investigation were determined by a nonlinear least-squares fit, and the resulting function generally provided a good fit to the local mean temperature rise data. In each of the figures 12,13 and 14, the profiles representing runs with more than $2 \% \mathrm{~F}_{2}$ were shifted in $y / \delta_{1}$ to coincide with the peak of the $2 \% \mathrm{~F}_{2}$ profiles to aid comparison. 


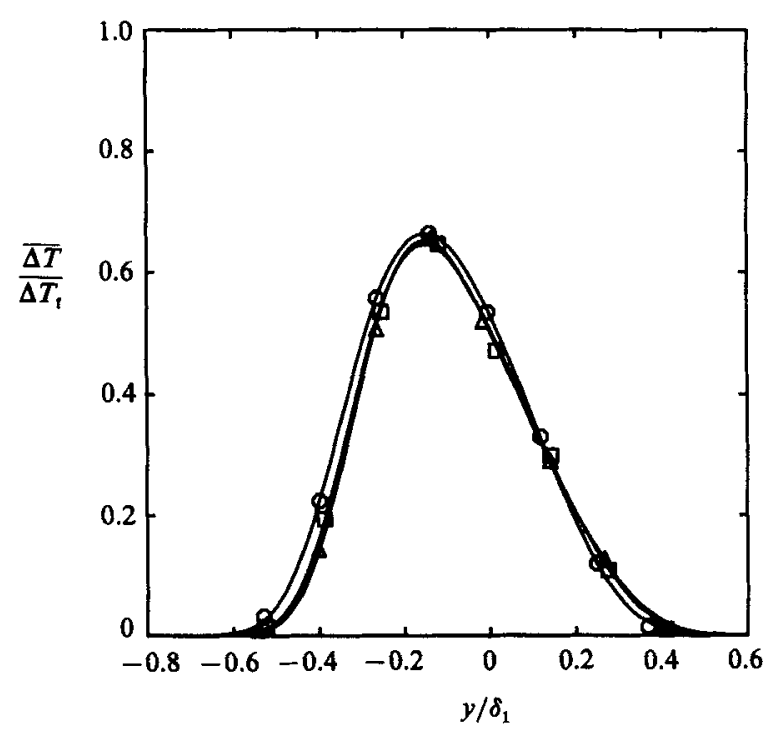

FIaURe 13. Mean temperature-rise profiles, $\phi=\frac{1}{2}: 0,2 \% \mathrm{~F}_{2}: 4 \% \mathrm{H}_{2}, \overline{\Delta \rho} / \rho_{0}=0.190 ; \square, 4 \% \mathrm{~F}_{2}$ : $8 \% \mathrm{H}_{2}, \overline{\Delta \rho} / \rho_{0}=0.292 ; \triangle, 6 \% \mathrm{~F}_{2}: 12 \% \mathrm{H}_{2}, \overline{\Delta \rho} / \rho_{0}=0.361$. Profiles shifted to coincide with peak of $2 \% \mathrm{~F}_{2}: 4 \% \mathrm{H}_{2}$ case.

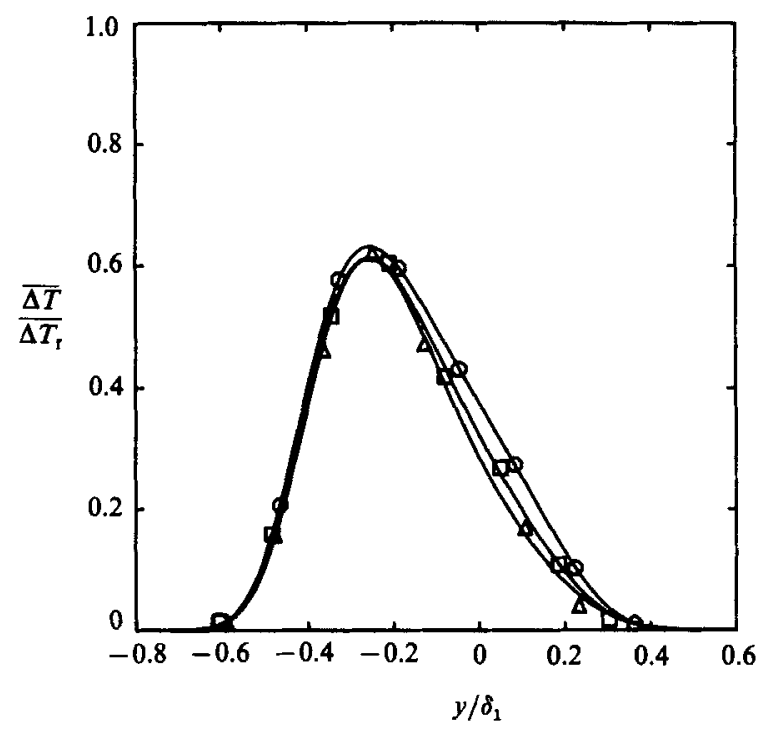

Froure 14. Mean temperature-rise profiles, $\phi=\frac{1}{4}: 0,2 \% \mathrm{~F}_{2}: 8 \% \mathrm{H}_{2}, \overline{\Delta \rho} / \rho_{0}=0.214 ; \square, 4 \% \mathrm{~F}_{2}$ : $16 \% \mathrm{H}_{2}, \overline{\Delta \rho} / \rho_{0}=0.311 ; \triangle, 6 \% \mathrm{~F}_{2}: 24 \% \mathrm{H}_{2}, \overline{\Delta \rho} / \rho_{0}=0.370$. Profiles shifted to coincide with peak of $2 \% \mathrm{~F}_{2}: 8 \% \mathrm{H}_{2}$ case.

An interesting result is that at a given stoichiometric mixture ratio the shape of the mean temperature profiles, normalized by the adiabatic flame temperatures, did not change greatly in spite of the large values of heat release. There appears to be no qualitative change in the profiles at $\phi=1$ and $\phi=\frac{1}{2}$. At $\phi=\frac{1}{4}$, a slight reduction in the profile height on the high-speed side can be observed.

Wallace (1981) and Mungal \& Dimotakis (1984) observed that the mean temperature at any point in the layer was substantially below the adiabatic flame 

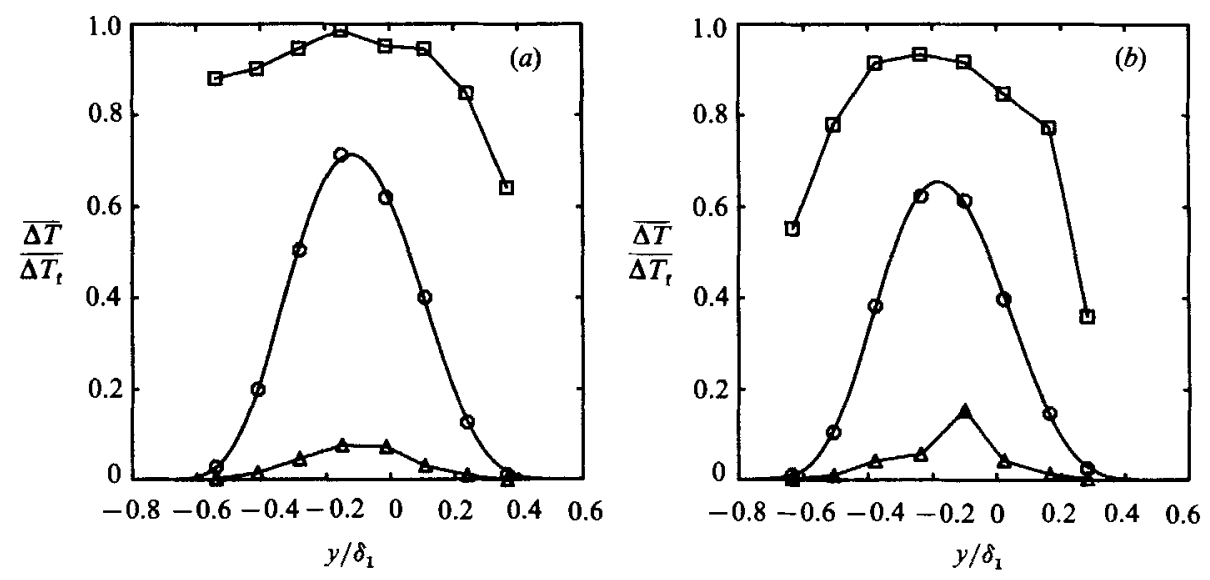

Frgure 15. Temperature-rise profiles with maxima and minima, $\phi=1: \bigcirc$, mean; $\square$, maximum; $\triangle$, minimum. (a) $4 \% \mathrm{~F}_{2}: 4 \% \mathrm{H}_{2}, \Delta T_{\mathrm{f}}=368 \mathrm{~K} ;(b) 6 \% \mathrm{~F}_{2}: 6 \% \mathrm{H}_{2}, \Delta T_{\mathrm{f}}=554 \mathrm{~K}$.

temperature. This was also observed in the present experiments at high heat release. This suggests that the mean temperature profiles, as at low temperature, result largely from a 'duty-cycle' phenomenon by which each probe spends a greater or lesser portion of the run time immersed in hot structure fluid, depending on its location in the layer, as proposed by Mungal \& Dimotakis (1984). This is consistent with the data shown in figure $11(a-c)$, where fairly distinct large, hot regions of fluid persist in the layer at elevated temperature. This gives a higher mean temperature near the centre of the layer and a lower value out near the layer edges. Employing an excess of high-speed reactant (i.e. $\phi<1$ ) results in a shift in the mean temperature-rise profile toward the side containing the lean reactant, consistent with the low-heat-release results of Wallace (1981) and Mungal \& Dimotakis (1984).

Konrad (1976) and Koochesfahani \& Dimotakis (1986) demonstrated that, in shear flows with no heat release, the composition of the mixed fluid is fairly uniform across the layer. This was also seen in the low-heat-release work of Mungal \& Dimotakis (1984) and appeared to persist, to some extent, in the present experiments. The extent to which the temperature in the large structures was uniform can be inferred by considering the maximum recorded temperatures at each point across the layer. The mean profiles at two selected values of heat release for $\phi=1$, and also the maximum and minimum temperatures recorded during the course of the entire run, are shown in figure $15(a, b)$. A similar comparison for $\phi=\frac{1}{4}$ is made in figure $16(a, b)$. In both figure sets, it can be seen that at lower temperatures the maximum temperatures in the central portions of the layer are relatively uniform, suggesting a fairly uniform temperature in the large structures. However, above about $\Delta T_{\mathrm{f}} \approx 500 \mathrm{~K}$, the maxima appear somewhat more peaked near the centre of the layer, suggesting that at higher temperatures the core regions of the structures become relatively hotter than the structure edges. As in Mungal \& Dimotakis (1984), none of these data were compensated for conduction error (see $\$ 2.2$ ), and one must assume that the probes were incapable of resolving the highest instantaneous temperature rise (near the adiabatic flame temperature rise), as well as the lowest instantaneous temperature rise (near zero for cold free-stream fluid).

It is possible to define a variety of parameters to quantify the trends in the amount of heat released and product formed. In the following discussion, two different 

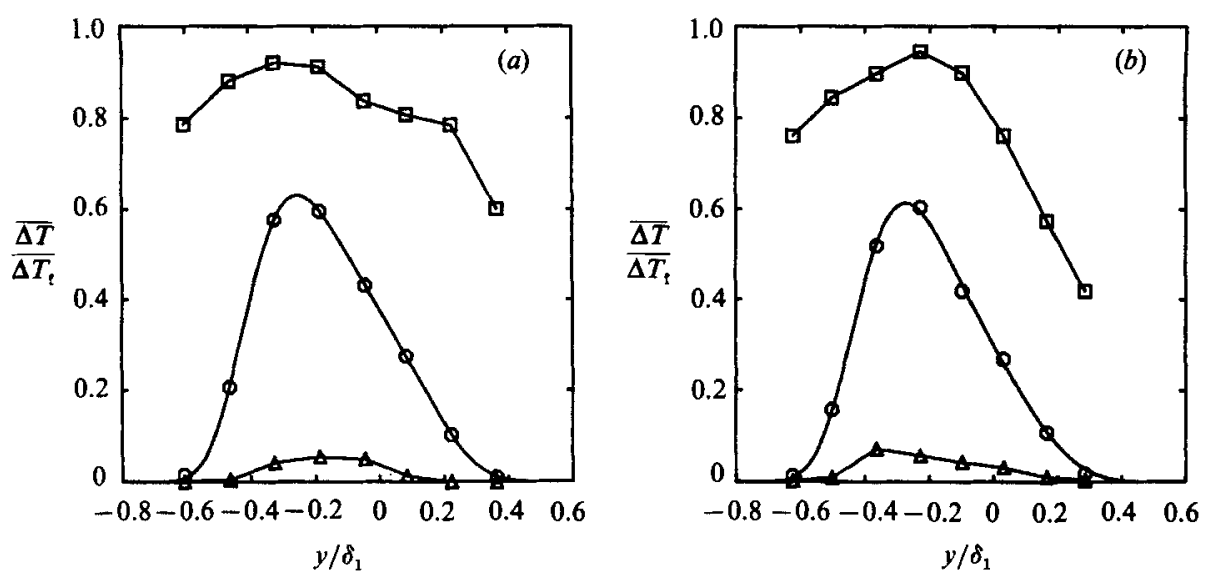

Figure 16. Temperature-rise profiles with maxima and minima, $\phi=\frac{1}{4}: O$, mean; $\square$, maximum; $\triangle$, minimum. (a) $2 \% \mathrm{~F}_{2}: 8 \% \mathrm{H}_{2}, \Delta T_{\mathrm{f}}=302 \mathrm{~K} ;(b) 4 \% \mathrm{~F}_{2}: 16 \% \mathrm{H}_{2}, \Delta T_{\mathrm{f}}=600 \mathrm{~K}$.

parameters will be employed for this purpose. The first, termed the 'product fraction thickness' in this work, is an integral measure which relates to the mole fraction of reactant converted to product in the mixing layer at a given downstream location. The second parameter is an integral measure of the total amount of product in the layer and will be referred to here as 'product mass thickness'.

The fractional conversion to chemical product can be related, for conditions of constant pressure, to the ratio of the enthalpy rise at a given measured temperature to the corresponding enthalpy rise indicated by the adiabatic flame temperature. Integration of the time-averaged value of this quantity across the width of the layer allows definition of the normalized 'product fraction thickness'

$$
\frac{\delta_{\mathrm{P}}}{\delta_{1}} \equiv \frac{1}{\delta_{1}} \int_{-\infty}^{\infty} \frac{\overline{\Delta H}(y)}{\Delta H\left(T_{\mathrm{f}}\right)} \mathrm{d} y .
$$

In this definition each value of the product fraction thickness, $\delta_{\mathrm{P}}$, is normalized by the corresponding local $1 \%$ width, $\delta_{1}$, which varies, as the case may be, for each value of heat release (as indicated in figure 3 ). In representing the mole fraction occupied by chemical product, (29) serves as a measure of the efficiency of the mixing and chemical reaction processes in the layer. Relating the enthalpy rise to the measured temperature rise allows writing

$$
\frac{\delta_{\mathrm{P}}}{\delta_{1}} \equiv \frac{1}{\delta_{1}} \int_{-\infty}^{\infty} \frac{\overline{\Delta T}(y)}{\Delta T_{\mathrm{f}}} \mathrm{d} y .
$$

This definition of normalized product fraction thickness was employed by Dimotakis (1987), and is identical to (29) in the case of low heat release where the mixture specific heat can be regarded as constant. Equation (30) was used in this investigation to calculate the values of normalized product fraction thickness. At the highest temperatures of the present study, the actual enthalpy of the gas mixture at the flame temperature differs from the value calculated using a constant specific heat and flame temperature rise by up to $8 \%$. Since the average flow temperature at any given location results from the passage of both hot and cold regions of gas, the actual error in representing $(29)$ by $(30)$ is less. 
The product fraction thickness defined by $(30)$ can be simply related to the normalized 'product thickness' used by Mungal \& Dimotakis (1984) to relate the temperature rise to the amount of product

$$
\frac{\delta_{\mathrm{P}_{2}}}{\delta_{1}} \equiv \frac{1}{\delta_{1}} \int_{-\infty}^{\infty} \frac{\hat{C}_{p} \overline{\Delta T}(y)}{c_{02} \Delta Q} \mathrm{~d} y,
$$

where $\hat{C}_{p}$ is the molar specific heat capacity at constant pressure and $\Delta Q$ is the molar heat release of the chemical reaction. If the specific heat is taken to be constant, and neglecting any effects of differential diffusion of heat or reactant species, the adiabatic flame temperature rise can be given as

$$
\Delta T_{\mathrm{f}}=\frac{1}{1+\phi} \frac{c_{02} \Delta Q}{\hat{C}_{p}}
$$

This corresponds to the case where $\phi$ is varied by keeping $c_{02}$ fixed and increasing $c_{01}$. Inserting the adiabatic flame temperature rise into (30) and comparing with (31) thus yields the relation $\delta_{\mathrm{P}}=(1+\phi) \delta_{\mathrm{P}_{2}}$.

An alternative definition for a product thickness based on the actual density of product can be obtained by integrating the local product fraction multiplied by the local fluid density, as follows

$$
\frac{\delta_{\mathrm{P}_{m}}}{\delta_{1}} \equiv \frac{1}{\delta_{1}} \int_{-\infty}^{\infty} \frac{\overline{\Delta T}(y)}{\Delta T_{\mathrm{f}}} \frac{\bar{\rho}(y)}{\rho_{0}} \mathrm{~d} y .
$$

The thickness defined by this relation will be termed the normalized 'product mass thickness'. The motivation for this alternative definition is to account for the decrease in the concentration of product, as the density in the layer decreases, owing to heat release. The earlier definition, the product fraction thickness defined in (30), could tend to a finite value even in the limit of infinite temperature rise (zero density) in the layer. The product mass thickness tends to the product fraction thickness in the limit of a very small temperature rise (i.e. small density changes). Relating the density to the local mean temperature gives

$$
\frac{\delta_{\mathrm{P}_{m}}}{\delta_{1}} \equiv \frac{1}{\delta_{1}} \frac{T_{0}}{\Delta T_{\mathrm{f}}} \int_{-\infty}^{\infty} \frac{\overline{\Delta T}(y)}{T_{0}+\overline{\Delta T}(y)} \mathrm{d} y .
$$

Relation (34) was used to compute the values of normalized product mass thickness.

Estimates of the product fraction thickness for this investigation are presented in figure 17. Some of the low-heat-release results calculated from the data of Mungal \& Dimotakis (1984) and Wallace (1981) are included for comparison. For the present investigation, some decrease in the product fraction thickness for all stoichiometric mixture ratios with heat release can be inferred, amounting to a reduction of approximately $18 \%$ for a mean density decrease of $24 \%$. By contrast, the data suggest an increase in product volume thickness with heat release between the lowheat-release results in this work and the results reported by Mungal \& Dimotakis (1984) and Wallace (1981). The relatively complicated behaviour of the product fraction thickness shown in figure 17 is the consequence of many competing physical mechanisms, which may result in temperature-induced changes in the volumetric entrainment ratio (see $\$ 5.3$ ), the intermittency, and the probability density of the mixed fluid. These issues are discussed in Hermanson (1985).

The results of the total product calculation suggested by the product mass fraction (34) are shown in figure 18. It is seen that the total amount of product (analogous also 


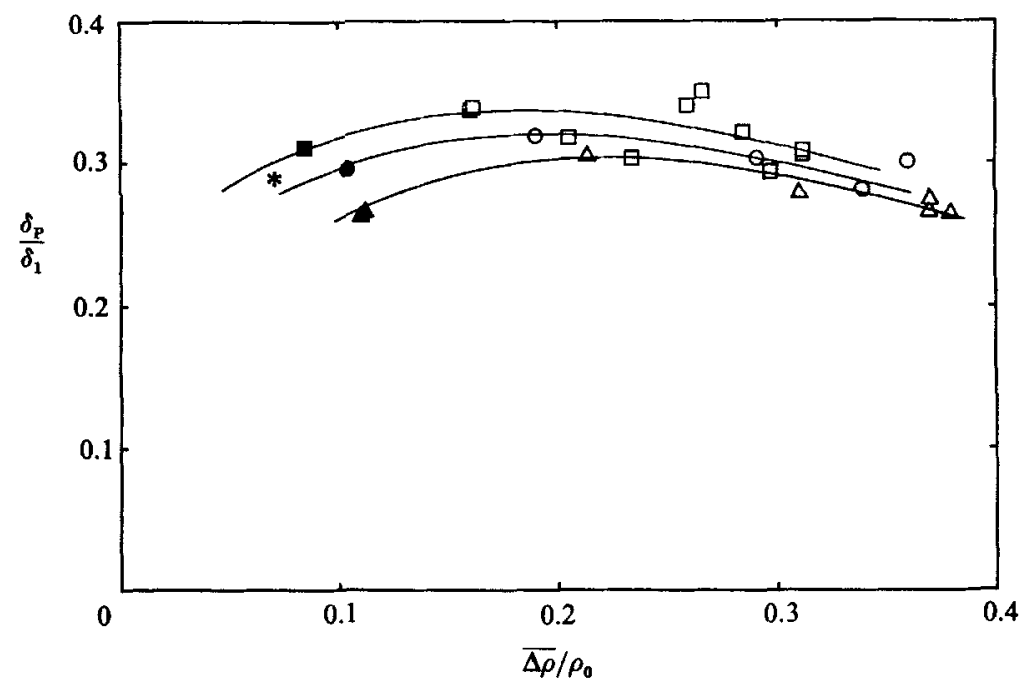

Figure 17. Product fraction thickness vs. heat release. $\square, \boldsymbol{\square}, *, \phi=1 ; 0, \boldsymbol{Q}, \phi=\frac{1}{2} ; \Delta, \Delta, \phi=\frac{1}{4}$. Solid symbols, Mungal \& Dimotakis (1984); open symbols, present work; asterisk, Wallace (1981).

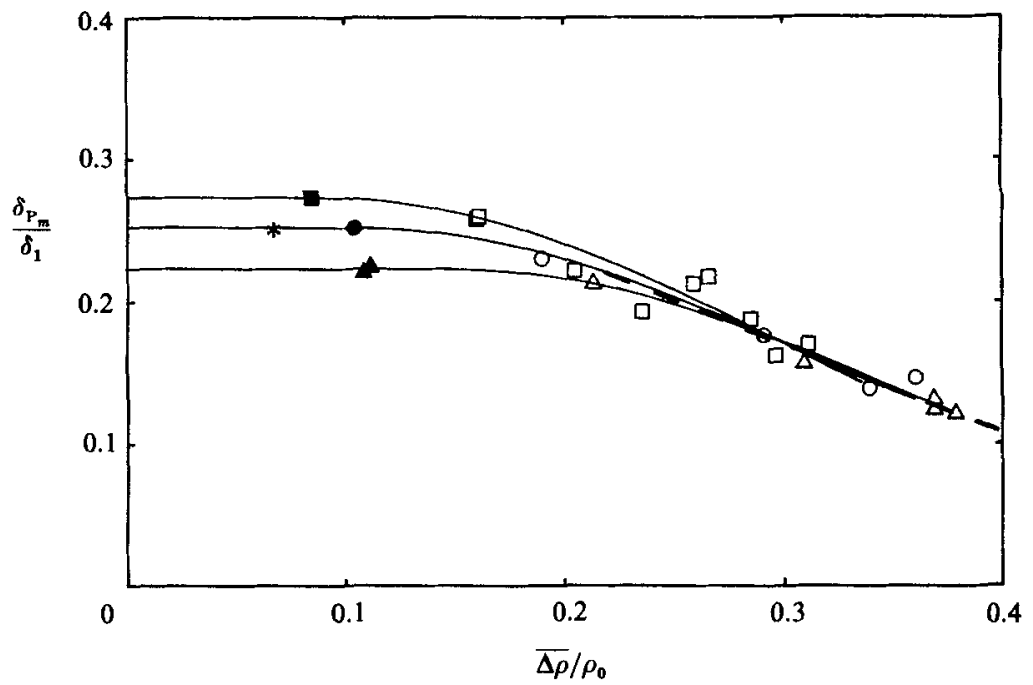

Figure 18. Product mass fraction thickness vs. heat release. $\square, \mathbf{\square}, \phi, \phi=1 ; 0, \boldsymbol{\theta}, \phi=\frac{1}{2} ; \Delta, \boldsymbol{\Delta}$, $\phi=\frac{1}{4}$. Solid symbols, Mungal \& Dimotakis (1984); open symbols, present work; asterisk, Wallace (1981). Dashed line is representative fit to data for all $\phi$ at high heat release.

to the total amount of heat release) decreases substantially with increasing temperature. This is partially due to the decrease in the product fraction in the layer at higher temperatures discussed previously, but appears to be mostly a consequence of the strong reduction owing to heat release in the entrainment of the reactants into the layer. It is also seen in figure 18 that while there is a noticeable dependence for the cases at low temperature rise on the amount of product with stoichiometric mixture ratio at a fixed level of heat release (i.e. fixed $\overline{\Delta \rho} / \rho_{0}$ ), this dependence appears to weaken considerably for higher values of heat release. In fact, beyond roughly $\overline{\Delta \rho} / \rho_{0}=0.2$, the product mass fraction data for all values of stoichiometric mixture ratio can apparently be represented by a single curve. 
(a)

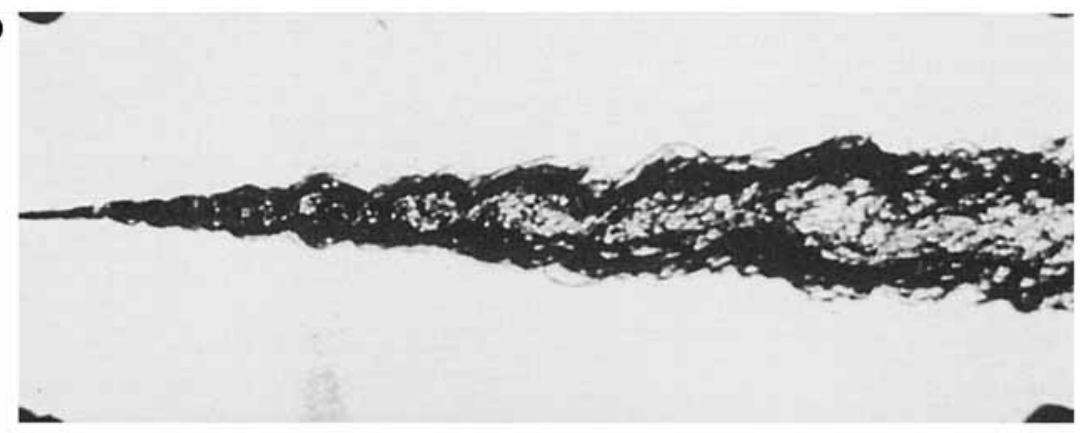

(b)

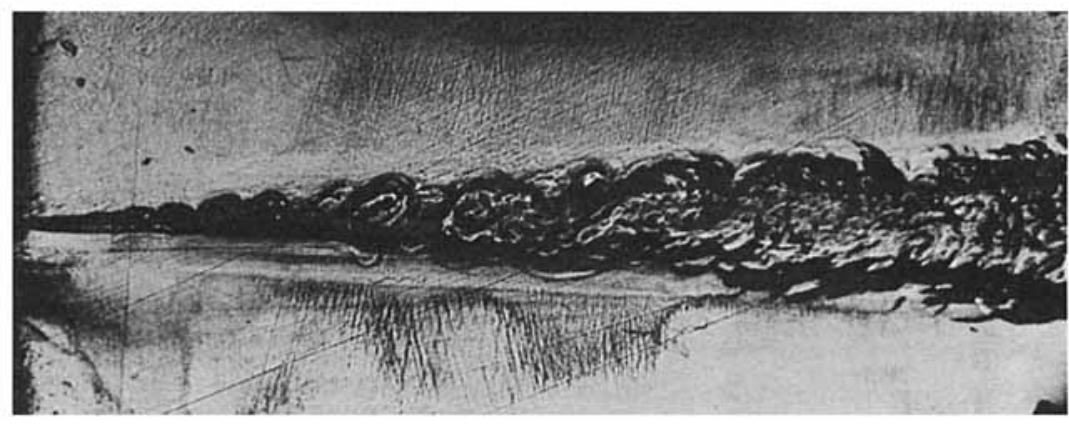

Figure 19. Spark schlieren photographs at low and high heat release. $(a) 2 \% \mathrm{~F}_{2}: 2 \% \mathrm{H}_{2}$, $\Delta T_{\mathrm{f}}=186 \mathrm{~K} ;\left(\right.$ b) $6 \% \mathrm{~F}_{2}: 16 \% \mathrm{H}_{2}, \Delta T_{\mathrm{f}}=847 \mathrm{~K}$.

The data in figure 18 may suggest a measure for determining some characteristic value of the heat-release parameter, $\overline{\Delta \rho} / \rho_{0}$, below which the effects of heat release can be ignored. Wallace (1981) suggests $\overline{\Delta T}_{\max } / T_{0}=0.35$ as the threshold of maximum mean temperature above which heat release ceases to be passive, as seen by a deviation of the measured maximum mean temperature from that predicted using the non-reacting probability density functions of Konrad (1976). The threshold value suggested by Wallace corresponds to $\overline{\Delta \rho} / \rho_{0} \approx 0.12$, which is roughly consistent with the speculative breaks in the curves shown in figure 18. This characteristic value could be defined as the break-point in each (constant- $\phi$ ) curve. Assuming that the curves in figure 18 tend to a constant value as $\overline{\Delta \rho} / \rho_{0}$ tends to zero suggests a possible means for extrapolating the product fraction thickness data, as presented in figure 17, to lower values of heat release. It should be remarked that the physical significance of the two product measure definitions used in this discussion cannot be regarded as being fully established, and that furthermore the trends shown in figures 17 and 18 may be, to some extent, artifacts of the definitions themselves.

\section{Large-scale structure dynamics}

The investigations of Wallace (1981) and Mungal \& Dimotakis (1984) showed that the large-scale structures that characterize non-reacting shear layers are also found in reacting layers at low heat release. Large-scale organization is also observable in shear flows at high temperatures, as shown by the work of Ganji \& Sawyer (1980), Pitz \& Daily (1983), and Keller \& Daily (1983). Large-scale structures were also observed at all values of heat release in this investigation. Sample spark schlieren photographs, corresponding to the lowest and to one of the highest levels of heat release in this investigation, are presented in figure $19(a, b)$. The schlieren sensitivity 
was reduced for the high-heat-release case, figure $19(b)$, from the low-heat-release run shown in figure $19(a)$, making qualitative comparison of the fine details between the two flows difficult; in both sample photographs, however, the large-scale structures can be clearly discerned. Ganji \& Sawyer (1980) argued that the schlieren visibility of the structures suggests that the large structures retain their predominately twodimensional nature even as the heat release increases.

In the present work, large-scale vortex statistical information was obtained over the full range of heat release from motion picture data. The location of the apparent centre of each identifiable vortex was digitized. The phenomenon of vortex amalgamation occasionally made identification of individual vortices difficult; in some frames it was difficult to pick out any large-scale structures. Ganji \& Sawyer (1980) pointed out that heat release might render the boundary between two merging structures invisible, while they might still be two separate fluid entities in the fluidmechanical sense. Nonetheless, a typical motion picture was digitized to provide, typically, about 1600 points corresponding to individual vortex locations. Normally about 1300 frames were digitized, corresponding to about $2.8 \mathrm{~s}$ of run time, during which over 300 large-scale structures were convected through the test section and field of view. Redigitization of several runs suggested that this technique produced mean statistics accurate to about $5 \%$. For cases with zero streamwise pressure gradient, little variation was observed with downstream distance in the local mean vortex spacing normalized by downstream distance and generally no significant difference in vortex statistics upstream and downstream of the mixing transition region.

Sample vortex-spacing histograms of four different runs, each with a different amount of heat release, are presented in figure $20(a-d)$. The histograms show the relative frequency of each value of vortex spacing normalized by the value of the $1 \%$ temperature thickness of the layer, $\delta_{1}$, at the station halfway between the two vortices in question, assuming linear layer growth. Increasing temperature appears to have caused a decrease in the mean normalized vortex spacing, $\bar{l} / \delta_{1}$. The corresponding standard deviation of the histograms, $\sigma$, also appears to have been reduced with heat release. The solid lines in figure $20(a-d)$ represent the lognormal distribution proposed by the statistical theory of vortex circulation formulated by Bernal (1981):

$$
P_{\lambda}(\lambda)=\frac{1}{(2 \pi)^{\frac{1}{2} \hat{\sigma} \lambda}} \exp \left[-\frac{1}{2 \hat{\sigma}^{2}}\left(\ln (\lambda / \bar{\lambda})+\frac{\hat{\sigma}^{2}}{2}\right)^{2}\right] .
$$

Here $\lambda \equiv l / \delta_{1}$, and the parameter $\hat{\sigma}$ is related to the variance of the experimentally determined distribution, $\sigma$, by

$$
\hat{\sigma}^{2}=\left(\frac{\bar{l}}{\delta_{1}}\right)^{2}\left(\mathrm{e}^{-\sigma^{2}}-1\right)
$$

The values of $\hat{\sigma}$ for the theoretical distributions shown in figure $20(a-d)$ were calculated using the $\bar{l} / \delta_{1}$ and $\sigma$ indicated by the experimental data.

The parameter $\hat{\sigma}$ in (35) was shown by Bernal to be related to the relative frequencies of vortex pairing, tripling and tearing. Bernal found a value of $\hat{\sigma}=0.28$ in the unreacting layer and showed that this value implies that pairing is the dominant vortex amalgamation mechanism. Increasing the relative frequency of tripling would result in a larger value of $\hat{\sigma}$; increasing tearing, a smaller value. In the present work, the values of $\hat{\sigma}$ calculated from the measured results were oserved to be nearly constant over the entire range of heat release, with a mean value of $\hat{\sigma}=0.25$. The near-constancy of $\hat{\sigma}$ suggests that the relative frequencies of pairing, 

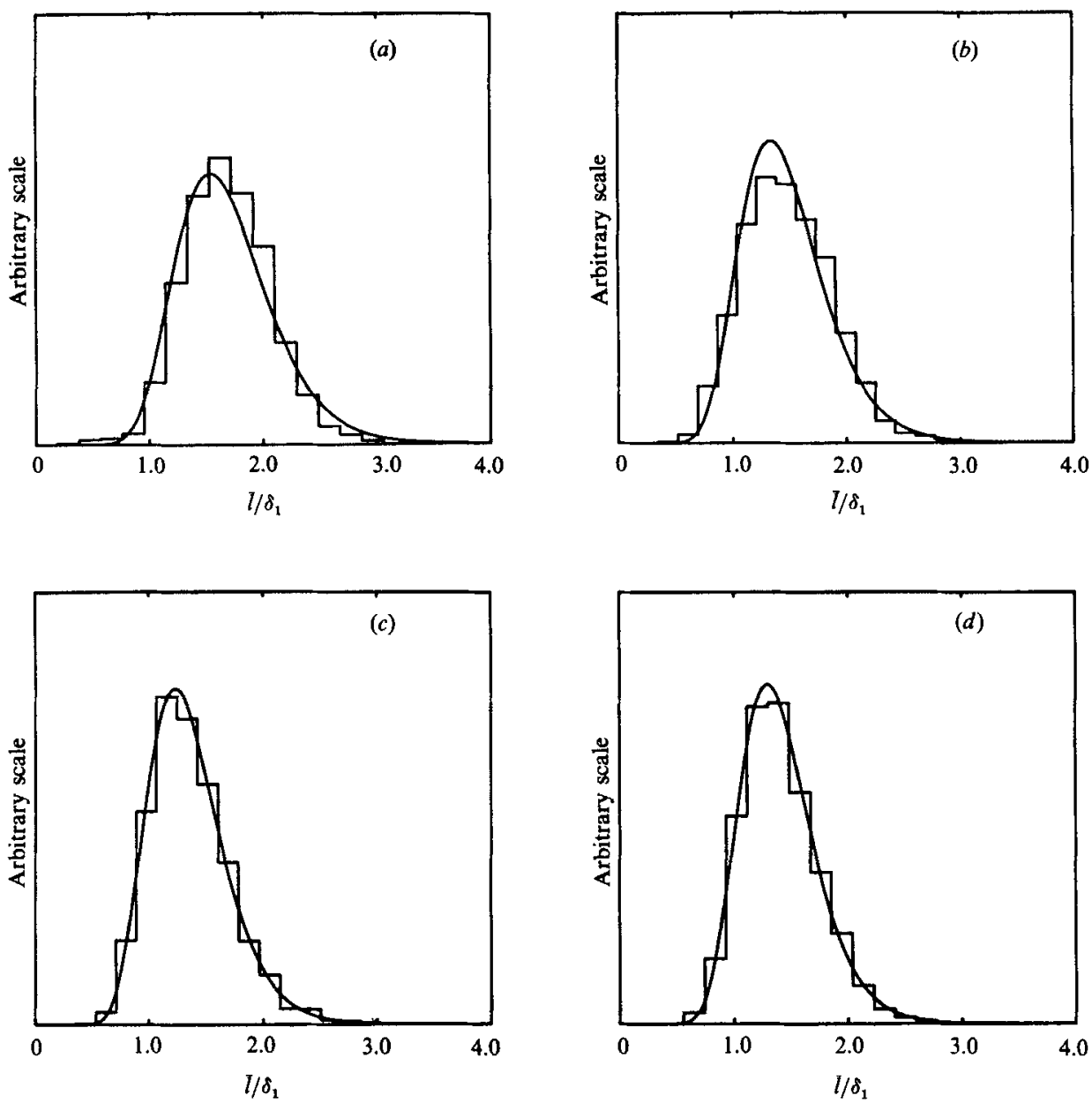

FIGURE 20. Vortex-spacing histograms. $(a) 2 \% \mathrm{~F}_{2}: 2 \% \mathrm{H}_{2}, \Delta T_{\mathrm{t}}=186 \mathrm{~K}, \bar{l} / \delta_{1}=1.69, \sigma=0.43$, $\hat{\sigma}=0.25 ;(b) \quad 4 \% \mathrm{~F}_{2}: 4 \% \mathrm{H}_{2}, \Delta T_{\mathrm{f}}=368 \mathrm{~K}, \bar{l} / \delta_{1}=1.48, \sigma=0.39, \hat{\sigma}=0.26 ;(c) \quad 6 \% \mathrm{~F}_{2}: 6 \% \mathrm{H}_{2}$, $\Delta T_{\mathrm{f}}=554 \mathrm{~K}, \bar{l} / \delta_{1}=1.36, \sigma=0.35, \hat{\sigma}=0.25 ;(d) 6 \% \mathrm{~F}_{2}: 12 \% \mathrm{H}_{2}, \bar{l} / \delta_{1}=1.25, \sigma=0.30, \hat{\sigma}=0.24$.

tripling, and tearing are not affected by heat release, though an increase (or decreast 3 ) of both tripling and tearing relative to pairing could also yield an unchanging valu te of $\hat{\sigma}$.

The decreasing trend in mean normalized vortex spacing, $\bar{l} / \delta_{1}$, with heat release is shown in figure 21 . The decrease was substantial, as large as $25 \%$ for a mean densit $y$ reduction of $40 \%$ in the layer. Because the vortex spacing decreased proportionall $y$ faster with heat release than the layer thickness, one could argue that the mechanisi $n$ of vortex amalgamation was inhibited, to some extent, by heat release.

Schlieren flow visualization in the non-reacting case in this experiment wo s accomplished by using nitrogen and a density-matched mixture of helium and argo $n$ as free-stream fluids. The measured mean normalized vortex spacing is in fair agreement with the corresponding results from Brown \& Roshko (1974), Bernal (1981), and Koochesfahani et al. (1979), which are included in figure $21 \mathrm{fcr}$ comparison.

The decrease in mean vortex spacing has implications for the volumetric 


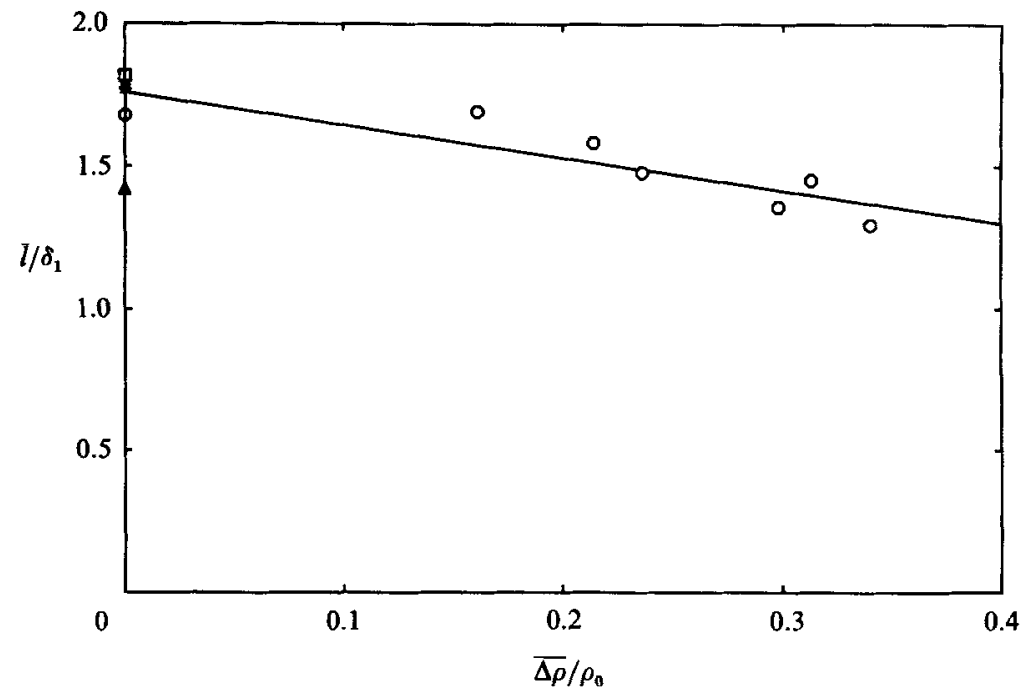

Figure 21. Mean vortex spacing vs. heat release: $\bigcirc$, present results; $\square$, Brown \& Roshko (1974); $\triangle$, Bernal (1981); *, Koochesfahani et al. (1979).

entrainment ratio into the layer. In Dimotakis (1986), the following expression is proposed for estimating the entrainment ratio:

$$
E_{v}=s^{\frac{1}{2}}\left(1+\frac{\bar{l}}{x}\right) .
$$

This implies that the reduction in vortex spacing is accompanied by a reduction in the volumetric entrainment ratio in favour of more entrained fluid from the lowspeed free stream. The observed decrease in mean vortex spacing indicates, using (37), a decrease in entrainment ratio of roughly $4 \%$ over the range of heat release in this investigation, which is within the range of entrainment ratio changes estimated in $\$ 5.3$.

The persistence of large-scale structures at elevated temperatures has an interesting implication for the mean density in a layer with heat release. The mean density reduction parameter, $\overline{\Delta \rho} / \rho_{0}$, is of course a function of the adiabatic flame temperature rise, $\Delta T_{\mathrm{f}}$. This dependence is shown for the present investigation in figure 22. Some results calculated from the data of Mungal \& Dimotakis (1984) and Wallace (1981) are also included. The mean density changed rapidly (with adiabatic flame temperature) at low heat release but less rapidly at high temperatures. These data suggest that there may be a limiting value of $\overline{\Delta \rho} / \rho_{0}$ at very high flame temperatures, and the present data suggest that the limiting value for a reacting shear layer is substantially less than unity. A limiting value of the mean density reduction might be expected if large-scale structures persist at all levels of heat release, resulting in the flow consisting of alternating regions of hot, low-density structure fluid and cold, high-density free-stream fluid. This would imply that even in the extreme case of infinitely high temperatures, where the cores of the structures would consist of fluid with essentially zero density, the regions of cold, unreacted free-stream fluid between structures would result in a finite mean density and a value of $\overline{\Delta \rho} / \rho_{0}$ less than unity.

The corresponding value of mean density reduction for the $U_{2}=0$ shear layer of 


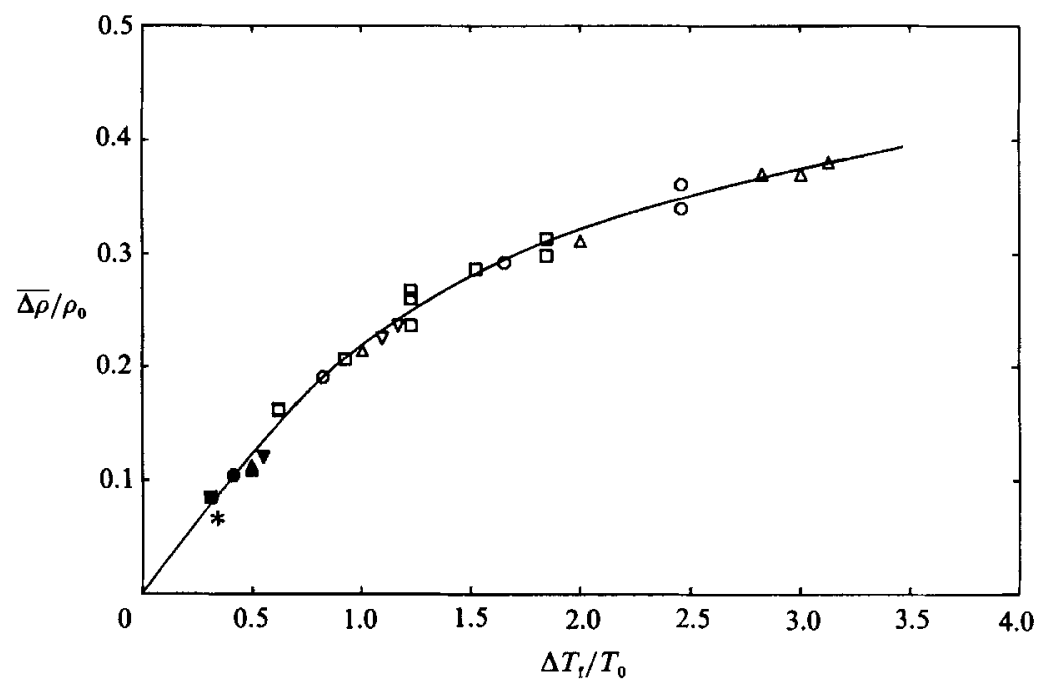

FigUre 22. Mean density reduction $v s$. adiabatic flame temperature rise: $\square, \boldsymbol{\square}, *, \phi=1 ; 0$ $\phi=\frac{1}{2} ; \triangle, \Delta, \phi=\frac{1}{4}$. Solid symbols: Mungal \& Dimotakis (1984); open symbols, present work; asterisk, Wallace (1981).

Ganji \& Sawyer $(1980)$ is $\overline{\Delta \rho} / \rho_{0} \approx 0.65$ for $\Delta T_{\mathrm{f}} / T_{0}=4.5$. It should be pointed out that their $U_{2}$ layer reattached and thus was characterized by the recirculation and re-entrainment of hot products into the low-speed side. This caveat may explain the difference between their results and the present data. On the other hand, their $U_{2} / U_{1} \approx 0$ shear-layer data may not necessarily be directly comparable to the $U_{2} / U_{1} \approx 0.4$ data presented here.

\section{Results for non-zero streamwise pressure gradient}

A pressure gradient in the present investigation was imposed by fixing the sidewalls for the reacting flow in a position corresponding to zero pressure gradient in the unreacting flow. This yielded a naturally induced favourable pressure gradient in the case of flow with chemical reaction, the magnitude of which increased in proportion to the level of heat release. The results with favourable pressure gradient, combined with those at zero pressure gradient discussed earlier allow comparison of flows with the same level of heat release but with different streamwise pressure gradients. In the present study, favourable pressure gradient runs were made at reactant concentrations of up to $6 \%$ fluorine and $6 \%$ hydrogen, corresponding to an adiabatic flame temperature rise of up to $554 \mathrm{~K}$. The amount of heat release was sufficient to induce a pressure decrease, $\Delta p$, over the distance from the splitter plate tip to the measuring station, of about half of $\frac{1}{2} \rho_{2} U_{2}^{2}$, the low-speed free-stream dynamic head. This served to increase the high-speed velocity from the nominal $22 \mathrm{~m} / \mathrm{s}$ to $23.1 \mathrm{~m} / \mathrm{s}$ and the low-speed velocity from $8.8 \mathrm{~m} / \mathrm{s}$ to $11.3 \mathrm{~m} / \mathrm{s}$ in the distance between the splitter tip and the measuring station, corresponding to a $22 \%$ decrease in the speed ratio, $U_{2} / U_{1}$.

\subsection{Shear-layer growth}

For a given level of heat release, the thickness of the layer, at a fixed measuring station, appeared to decrease for each favourable-pressure-gradient case, relative to 
the corresponding flow with no streamwise pressure gradient. The thinning caused by pressure gradient is not surprising in an accelerating flow. In particular, the change in layer growth rate can be accounted for in terms of the change with downstream distance in speed ratio induced by the pressure gradient, as quantified in the following discussion.

For non-reacting shear layers with equal free-stream densities, a commonly used expression for relating the growth rate to the speed ratio was given by Brown \& Roshko (1974) (this is a special case of the more general form of (4), §5.1):

$$
\frac{\delta_{\text {vis }}}{\left(x-x_{0}\right)}=0.38 \frac{1-r}{1+r}
$$

where $\delta_{\text {vis }}$ is the visual thickness of the layer. For the moderate values of the streamwise pressure gradient in this investigation, it is interesting to take this similarity relation as holding locally†, i.e.

$$
\frac{\mathrm{d} \delta_{1}}{\mathrm{~d} x}=\Lambda(\hat{q}) \frac{1-r(x)}{1+r(x)},
$$

where the parameter $A(\hat{q})$ is a growth-rate parameter, taken to depend only on the amount of heat release. The speed ratio, $r(x) \equiv U_{2}(x) / U_{1}(x)$, is a local function of the downstream distance in the presence of a pressure gradient. Thus in this formation the growth rate is expressed as a product of two factors, one that relates only to the heat release, the other that depends on the magnitude of the pressure gradient. Equation (39) is essentially a statement that the layer is in local equilibrium, that is, that the speed ratio changes slowly compared with the rate at which the layer growth adjusts to it. A changing speed ratio with downstream distance in (39) due to pressure gradient thus indicates a nonlinear growth rate. It should be remarked that the speed ratio appears to impact the mean vortex spacing normalized by the layer thickness, as shown by the results of Browand \& Troutt (1985). This effect is not strong, however, and could be expected for the largest amount of pressure gradient discussed here to result in a normalized vortex spacing change of about $1 \%$. This should be compared to the observed variation in layer thickness with pressure gradient of up to $8 \%$. The change in normalized vortex spacing is not directly addressed by the present analysis.

Integration of (39) gives the layer growth as a function of downstream distance, i.e.

$$
\delta_{1}(x)=\Lambda(\hat{q}) \int_{0}^{x} \frac{1-r(x)}{1+r(x)} \mathrm{d} x .
$$

From (40) the thickness of a layer with heat release but at zero pressure gradient, $\delta_{1_{0}}(x)$, is

$$
\delta_{1_{0}}(x)=\Lambda(\hat{q}) \frac{1-r_{0}}{1+r_{0}} x
$$

where $r(x) \equiv r_{0}=$ constant in the absence of a pressure gradient. Consideration of two layers at the same level of heat release (i.e. same value of $\Lambda(\hat{q}))$ but with and without pressure gradient allows normalization of (40) by (41) to give

$$
\frac{\delta_{1}(x)}{\delta_{1_{0}}(x)}=\frac{1}{x} \frac{1+r_{0}}{1-r_{0}} \int_{0}^{x} \frac{1-r(x)}{1+r(x)} \mathrm{d} x .
$$

$\dagger$ Suggested by M. M. Koochesfahani in private discussions. 


$\begin{array}{cccccc}\% \mathrm{~F}_{2} / \% \mathrm{H}_{2} & \Delta T_{\mathrm{t}}(\mathrm{K}) & \overline{\Delta \rho} / \rho_{0} & \frac{\mathrm{d} p}{\mathrm{~d} x}(\text { torr } / \mathrm{cm}) & \kappa(\hat{q})\left(\mathrm{cm}^{-1}\right) & \delta_{1}(\mathrm{~cm}) \\ - & & & & & \\ 2 / 2 & 186.3 & 0.153 & \approx 0 & \approx 0 & 7.13 \\ 2 / 2 & 186.3 & 0.156 & -0.0022 & -0.0027 & 7.00 \\ 4 / 4 & 368.2 & 0.260 & \approx 0 & \approx 0 & 7.04 \\ 4 / 4 & 368.2 & 0.257 & -0.0032 & -0.0053 & 6.49 \\ 6 / 6 & 553.8 & 0.313 & \approx 0 & \approx 0 & 6.72 \\ 6 / 6 & 553.8 & 0.312 & -0.0042 & -0.0080 & 6.45\end{array}$

TABLE 2. Representative pairs of runs without and with streamwise pressure gradient. $\mathrm{d} p / \mathrm{d} x \equiv$ pressure gradient; $\kappa(\hat{q}) \equiv$ pressure gradient parameter (see text) $; \delta_{1} \equiv$ layer thickness at measuring station based on $1 \%$ points of mean temperature rise profile. Flows at nominally zero pressure gradient have values of $\kappa(\hat{q})< \pm 0.0003 \mathrm{~cm}^{-1}$.

The lower limit of integration in (40) and (42) is taken as $x=0$; this analysis thus does not explicitly consider the effects of a virtual origin, $x_{0}$. By neglecting the virtual origin, it is essentially assumed that both the layer with a pressure gradient and the corresponding layer at zero pressure gradient attain the growth rate suggested by (39) in a downstream distance that is small compared with the distance from the splitter tip to the measuring station. In \$5.1 the virtual origin for the zero-pressuregradient case was seen to be typically about $7 \%$ of the total distance to the measuring station. It should be noted that the layer in this investigation was not selfsimilar in the presence of a streamwise pressure gradient. This conclusion follows from the work of Rebollo (1973), who showed that, in order to have similarity, the layer growth must be linear and the free-stream dynamic heads must be matched. Neither condition was met in the present work.

The free-stream velocities can be determined from the corresponding Bernoulli equations, assuming that the streamwise pressure gradient $\mathrm{d} p / \mathrm{d} x$ does not depend on downstream distance for the particular flow in question. The results are

and

$$
\begin{gathered}
U_{1}^{2}(x)=U_{01}^{2}-\frac{2}{\rho_{1}}\left[\frac{\mathrm{d} p}{\mathrm{~d} x}\right] x \\
U_{2}^{2}(x)=U_{02}^{2}-\frac{2}{\rho_{2}}\left[\frac{\mathrm{d} p}{\mathrm{~d} x}\right] x,
\end{gathered}
$$

where $U_{01}$ and $U_{02}$ are the high-speed and low-speed free-stream velocities, respectively, at some reference point, e.g. at the start of the test section $(x=0)$. The values of $\mathrm{d} p / \mathrm{d} x$ and the observed layer thickness at the measuring station are given in table 2 for several values of heat release.

The speed ratio as a function of downstream distance can be expressed by combining $(43 a)$ and $(43 b)$. The result is

$$
\begin{gathered}
r(x)=r_{0}\left[\frac{1-2 \kappa(\hat{q}) x}{1-2 \kappa(\hat{q}) r_{0}^{2} s x}\right]^{\frac{1}{2}}, \\
\kappa(\hat{q}) \equiv \frac{\mathrm{d} p / \mathrm{d} x}{\rho_{2} U_{02}^{2}}
\end{gathered}
$$

where

is introduced here as the pressure gradient parameter, which has units of inverse length, and $r_{0} \equiv U_{02} / U_{01}$ is the initial velocity ratio (at $x=0$ ). For the fixed geometry in this work, $\kappa(\hat{q})$ depends directly on the level of heat release employed. The product 


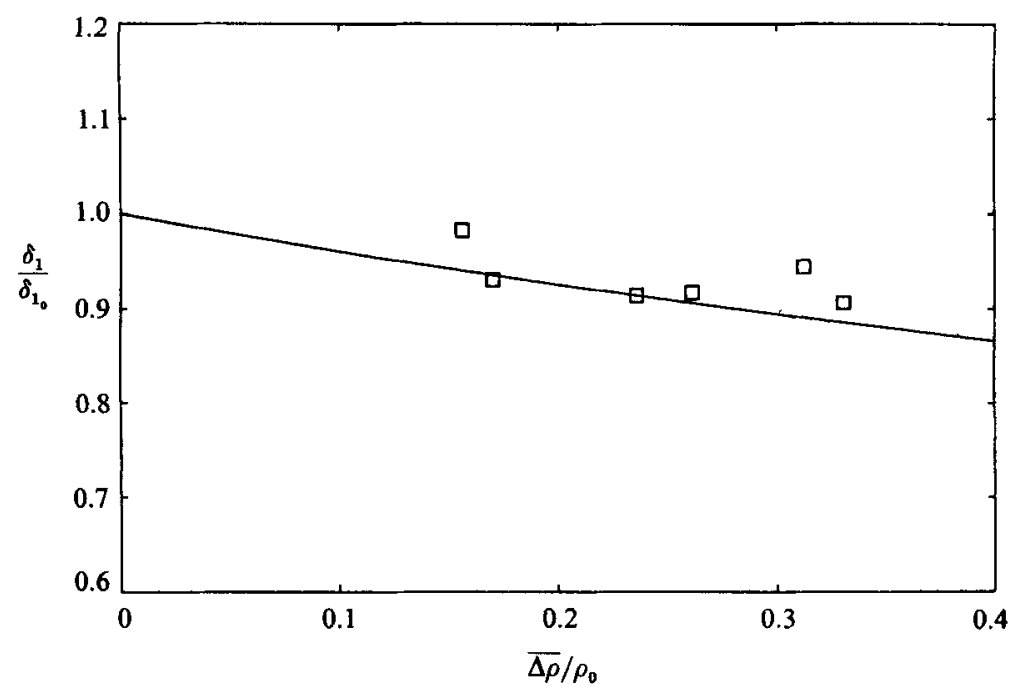

Frgure 23. Normalized layer thickness with streamwise pressure gradient: - 一, theory; $\square$, experiment. $\delta_{1_{0}}$ is layer width at given level of heat release with no pressure gradient.

$\kappa(q) x$ is dimensionless and can be thought of as characterizing the cumulative effect of the pressure gradient over the length $x$. A non-dimensional parameter similar to $\kappa(\hat{q}) x$,

$$
\beta \equiv \frac{d_{\mathrm{j}}}{\rho_{\mathrm{j}} u_{\mathrm{j}}^{2}} \frac{\mathrm{d} p}{\mathrm{~d} x},
$$

was presented by Stårner \& Bilger (1980) as a means of parameterizing reacting jet flows with streamwise pressure gradient. Here $d_{\mathrm{j}}$ is the jet diameter, $\rho_{\mathrm{j}}$ and $u_{\mathrm{j}}$ the jet fluid density and velocity, respectively.

The effect of a favourable pressure gradient is seen from (44) to increase the speed ratio (i.e. drive it toward unity); and to decrease it (drive it toward zero) for an adverse pressure gradient. The speed ratio is affected less by a favourable pressure gradient than by an adverse one of similar magnitude.

The trend in layer thinning over the full range of heat release and pressure gradient of this investigation is shown in figure 23 , where the normalized layer thickness $\left(\delta_{1} / \delta_{1_{0}}\right)$ at the measuring station is given against $\overline{\Delta \rho} / \rho_{0}$. The curve shown results from (42) and (44). In this calculation the values of $\mathrm{d} p / \mathrm{d} x$ versus $\overline{\Delta \rho} / \rho_{0}$ were determined by a linear least-squares fit to the experimental data. The fit was on average accurate to about $6 \%$. Values of $r_{0}=0.38$ and $s=1$ were representative of the cases at zero pressure gradient considered here and were employed in the calculation.

Figure 23 thus indicates how the layer thickness for a given amount of heat release with the pressure gradient induced by the fixed wall locations of this experiment compares with the corresponding layer with zero streamwise pressure gradient. The experimental results are seen to be consistent with the predicted trend. The above analysis appears to adequately represent the layer growth and the changes in the free-stream conditions for the values of heat release and pressure gradient in this investigation. It is not clear whether these arguments will continue to be valid for larger values of favourable pressure gradient and higher flow temperatures, or for adverse pressure gradients.

The mean velocity profiles at the measuring station, other than showing a change 


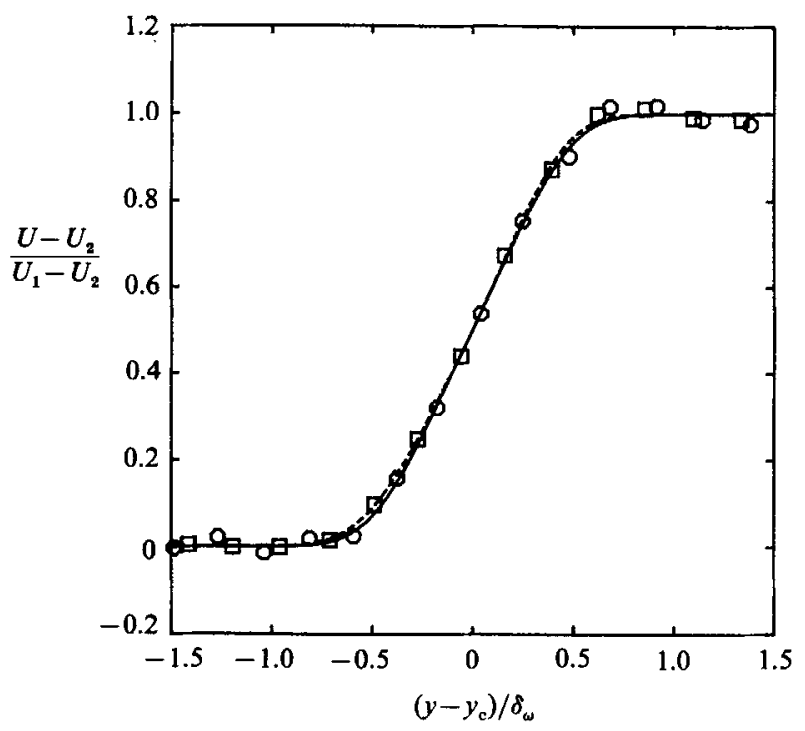

Fraure 24. Mean velocity profile comparison, $6 \% \mathrm{~F}_{2}: 6 \% \mathrm{H}_{2}, \Delta T_{\mathrm{f}}=554 \mathrm{~K} . \mathrm{O}, \kappa(\hat{q}) \approx 0$, $\delta_{\omega} /\left(x-x_{0}\right)=0.072 ; \square, \kappa(\hat{q})=-0.0080 / \mathrm{cm}, \delta_{\omega} /\left(x-x_{0}\right)=0.068$.

in maximum slope corresponding to a change in layer thickness, did not exhibit any significant differences in shape between the corresponding cases with and without pressure gradient. The normalized velocity profile for the maximum amount of pressure gradient in this investigation is compared with the corresponding profile at zero pressure gradient in figure 24 .

\subsection{Temperature rise and amount of product formation}

It could be argued that, in reacting flows with substantial density variations and appreciable pressure gradients, an additional mixing mechanism might be operative, resulting from a possible relative acceleration between light fluid elements and heavy fluid elements. This mechanism is referred to as 'pressure gradient diffusion' by Bray \& Libby (1981) and by Spalding (1986). The efficacy of such a mechanism would of course depend on the scales at which the density variations would occur and their relation to the viscous small scales of the flow. If the hot/cold fluid elements are very closely interlaced, viscous effects might not permit substantial relative motions to be established and little or no augmentation of the mixing would result.

The results of this experiment indicate little change in either the peak temperature or the amount of product formation as a result of the favourable pressure gradient. This is seen in figure 25, where three of the resulting mean temperature profiles, at the highest heat release and largest value of pressure gradient attained in this investigation, are compared. The profiles for $\kappa(\hat{q})<0$ were shifted as in figure 12 to aid comparison. These results indicate a maximum possible augmentation in product, as indicated by the product volume fraction defined in $\$ 6$, of about $8 \%$. The cases with pressure gradient were seen to be, for all values of heat release, on average less than $5 \%$ higher in product thickness than the corresponding flows without pressure gradient. Bearing in mind that the estimated accuracy of the experimental results is $3-5 \%$, these data also admit the possibility that the effect may be considerably smaller.

A small increase in product can be accounted for by the change in Reynolds 


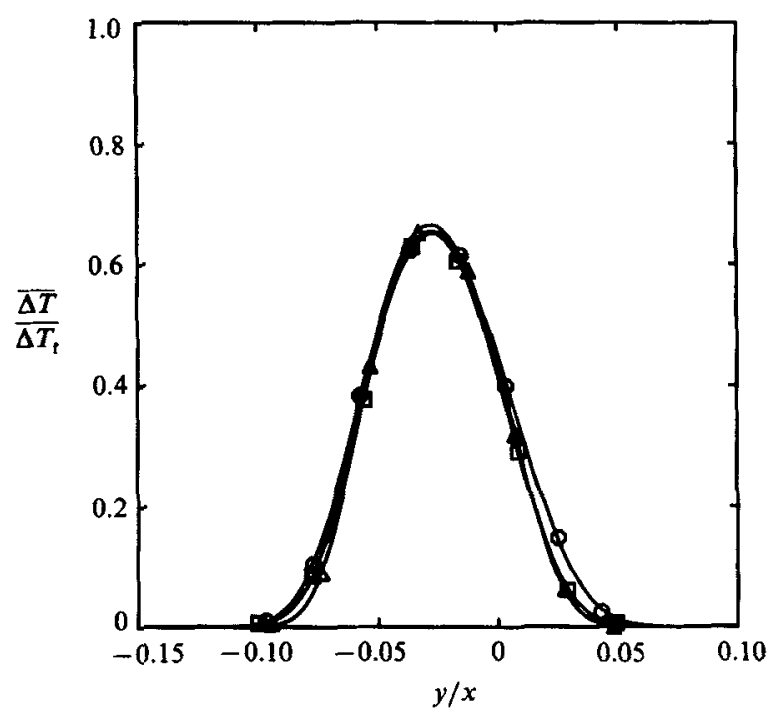

Figure 25. Pressure gradient effect on mean temperature profile, $6 \% \mathrm{~F}_{2}: 6 \% \mathrm{H}_{2}, \Delta T_{\mathrm{f}}=554 \mathrm{~K}$. $\mathrm{O}, \kappa(\hat{q}) \approx 0 ; \square, \kappa(\hat{q})=-0.0080 / \mathrm{cm} ; \Delta, \kappa(\hat{q})=-0.0088 / \mathrm{cm}$. Profiles shifted to coincide with peak of $k(\hat{q})=0$ case.

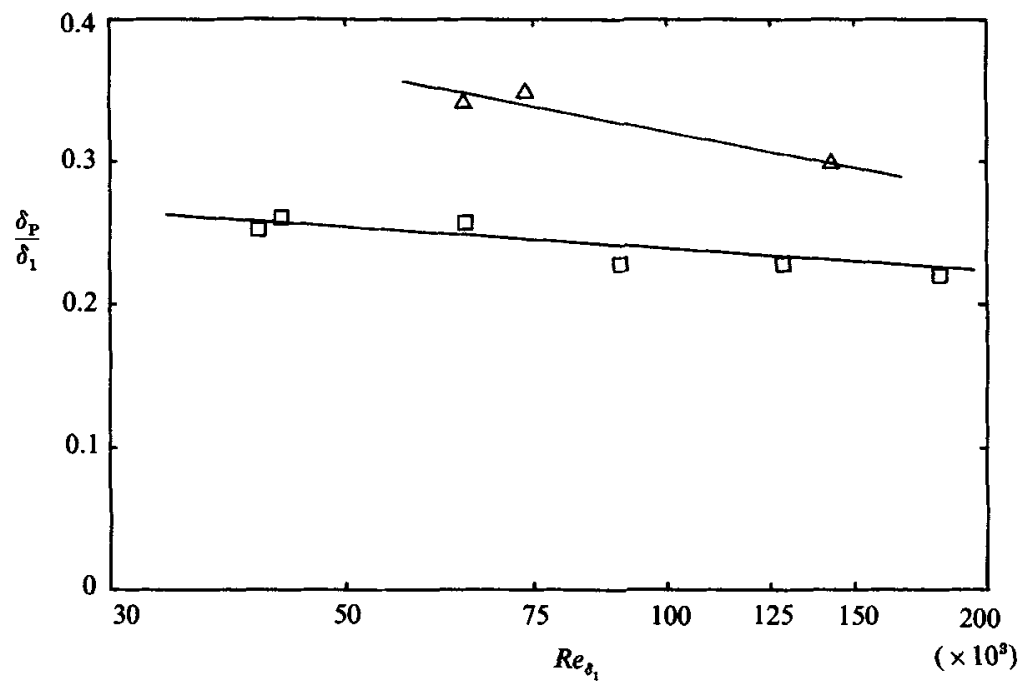

FIGURE 26. Reynolds-number effect on product volume thickness: $\square$, Mungal et al. (1985) $\phi=\frac{1}{8}$, $\Delta T_{1}=186 \mathrm{~K} ; \Delta$, present results, $\phi=1, \Delta T_{\mathrm{f}}=368 \mathrm{~K}$.

number of the flow caused by pressure gradient. The local value of Reynolds number, $R e_{\delta_{1}} \equiv \Delta U \delta_{1} / \nu$, at a given downstream location, decreases with a favourable pressure gradient. This is because $\delta_{1}$ decreases and, in addition, $\Delta U$ decreases owing to freestream acceleration. The result is a modest decrease in Reynolds number. The nominal Reynolds number at the measuring station of $R e_{\delta_{1}} \approx 6.4 \times 10^{4}$ decreased to as little as $R e_{\delta_{1}} \approx 5.2 \times 10^{4}$, for the runs with the largest heat release and pressure gradient.

How the amount of product in the layer depends on the Reynolds number was investigated at low heat release by Mungal et al. (1985). That work suggested an 
increase in product formation amounting to roughly $20 \%$ per factor of 10 decrease in Reynolds number. How that trend might change with heat release was investigated by running $4 \%$ fluorine and $4 \%$ hydrogen $\left(\Delta T_{\mathrm{f}}=368 \mathrm{~K}\right)$ at elevated free-stream speeds, $U_{1}=44 \mathrm{~m} / \mathrm{s}, U_{2}=17.6 \mathrm{~m} / \mathrm{s}$. The results of these runs, along with those of Mungal et al. are shown in figure 26. The trend of product decrease with Reynolds number at low heat release appears to persist at higher values of heat release.

The data in figure 26 suggest, for the present investigation, that the naturally induced pressure gradient could be expected to result in an increase in product formation of up to approximately $5 \%$ at the highest temperature and pressure gradient of this investigation. That this result already accounts for most of the small observed increase in product formation suggests that 'pressure gradient diffusion', at least for the values of temperature and pressure gradient reported here, does not play a significant role in enhancing the mixing and combustion in a reacting shear layer.

\section{Conclusion}

The hydrogen-fluorine chemical reaction was used to study the effects of large heat release in a planar, turbulent shear layer at high Reynolds number. A range of reactant concentrations was employed to produce adiabatic flame temperature rises from $186 \mathrm{~K}$ up to $940 \mathrm{~K}$, resulting in a maximum mean density reduction in the layer of up to $60 \%$.

The growth rate of the shear layer, in spite of large heat release and large density changes, did not increase and instead appeared to show a slight decrease, even as the displacement thickness of the layer increased substantially with heat release. The decrease in growth rate, at zero streamwise pressure gradient, was observed to be up to $15 \%$ for a mean density reduction in the layer of $40 \%$. Accordingly the volumetric entrainment of free-stream fluids into the layer was substantially reduced by heat release. The observed reduction in entrainment appears to more than offset the additional displacement of the layer owing to heat release. $E x$ post facto arguments suggest that a large decrease in turbulent momentum transport, as represented by the turbulent shear stress in the layer, accompanies increasing flow temperatures. This decrease in shear stress can be accounted for by the change in density in the core regions of the large-scale structures, and is shown to be consistent with the reduced layer growth.

The mean temperature-rise profiles, normalized by the adiabatic flame temperature, did not change significantly in shape over the entire range of heat release investigated. A slight decrease in normalized mean temperature was observed, which indicates that the relative efficiency with which the layer generates chemical product decreases slightly with increasing heat release. This further suggests that the probability density function of the mixed-fluid concentration is altered, to some extent, by heat release.

Large-scale structures persisted in the shear layer at all levels of heat release. The mean structure spacing, normalized by the layer width, decreased with increasing heat release. This suggests that the mechanisms of large-structure coalescence are, to some extent, inhibited by heat release.

The imposition of a favourable pressure gradient resulted in additional layer thinning at all levels of heat release. This change in growth rate can be related to the change in free-stream speed ratio induced by the favourable pressure gradient. The pressure gradient had little effect on the amount of mixing and chemical product 
formation in the layer. The slight measured increase in product appears to be largely accounted for by the decrease in the local Reynolds number resulting from the acceleration of the free-streams and the layer thinning. The increase in the amount of product with decreasing Reynolds number is in accord with earlier results obtained at low heat release.

The authors gratefully acknowledge the expert advice and assistance of Dr M. G. Mungal as well as the help of C. E. Frieler, Dr R. C. Miake-Lye, and Mr Earl Dahl in running the experiments reported here. We are also thankful for the helpful input and ideas of Drs J. E. Broadwell and M. M. Koochesfahani. This work was sponsored by the Air Force Office of Scientific Research (AFOSR) Contract No. F49620-79-C0159 and Grant No. 83-0213.

\section{REFERENCES}

BAtT, R. G. 1975 Some measurements on the effect of tripping the two-dimensional shear layer. AIAA J. 13, 245-247.

Baulch, D. L., Duxbury, J., Grant, S. J. \& Montague, D. C. 1981 Evaluated kinetic data for high temperature reactions, vol. 4. J. Phys. Chem. Ref. Data, 10, Suppl. 1.

Bernal, L. P. 1981 The coherent structure of turbulent mixing layers: I. Similarity of the primary vortex structure. II. Secondary streamwise vortex structure. Ph.D. thesis, California Institute of Technology.

Bernal, L. P., Breidenthal, R. E., Brown, G. L., Konrad, J. H. \& Roshro, A. 1979 On the development of three-dimensional small scales in turbulent mixing layers. In Turbulent Shear Flows 2, Seond Intl Symp. on Turbulent Shear Flows, July 1979, p. 305. Springer.

Bradshaw, P. 1966 The effect of initial conditions of the development of a free shear layer. J. Fluid Mech. 26, 225-236.

Bray, K. N. C. \& LibBy, P. A. 1981 Countergradient diffusion in pre-mixed turbulent flames, AIAA J. 19, 205-213.

BReIDen'thaL, R. E. 1981 Structure in turbulent mixing layers and wakes using a chemical reaction. J. Fluid Mech. 109, 1-24.

Broadwell, J. E. \& Breidenthal, R. E. 1982 A simple model of mixing and chemical reaction in a turbulent shear layer. J. Fluid Mech. 125, 397-410.

Browand, F. K. \& Latigo, B. O. 1979 Growth of the two-dimensional mixing layer from a turbulent and non-turbulent boundary layer. Phys. Fluids 22, 1011-1019.

Browand, F. K. \& TroutT, T. R. 1985 The turbulent mixing layer: geometry of large vortices. J. Fluid Mech. 158, 489-509.

Brown, G. L. \& Roshko, A. 1971 The effect of density differences on the turbulent mixing layer. In Turbulent Shear Flows; AGARD-CP-93, 23.1-23.12.

Brown, G. L. \& Roshko, A. 1974 On density effects and large structure in turbulent mixing layers. J. Fluid Mech. 64, 775-816.

Brown, J. L. 1978 Heterogeneous turbulent mixing layer investigations utilizing a 2-D 2-color laser Doppler anemometer and using a concentration probe. Ph.D. dissertation, University of Missouri at Columbia.

Caldwell, F. R. 1962 Thermocouple materials. National Bureau of Standards Monograph 40. United States Department of Commerce, National Bureau of Standards.

CoHen, N. \& BoTr, J. F. 1982 Review of rate data for reactions of interest in HF and DF lasers. The Aerospace Corporation Rep. SD-TR-82-86.

Dimotakis, P. E. 1986 Entrainment and growth of a fully developed, two-dimensional shear layer. $A I A A$ J. 24, 1791-1796.

Dimotakis, P.E. 1987 Turbulent shear layer mixing with fast chemical reactions. United States-France Joint Workshop on Turbulent Reacting Flows, Rouen, France, July 1987 (submitted to J. Fluid Mech). 
Dimotakis, P. E. \& Brown, G. L. 1976 The mixing layer at high Reynolds number: largestructure dynamics and entrainment. J. Fluid Mech. 78, 535-560.

Effelsberg, E. \& Peters, N. 1983 A composite model for the conserved scalar PDF. Combust. Flame 50, 351-360.

Ganj, A. T. \& SAwyer, R. F. 1980 Experimental study of the flowfield of a two-dimensional, premixed turbulent flame. AIAA J. 18, 817-824.

Hermanson, J. C. 1985 Heat release effects in a turbulent, reacting shear layer. Ph.D. thesis, California Institute of Technology.

Hermanson, J. C., Mungal, M. G. \& Dimotakis, P. E. 1987 Heat release effects on shear layer growth and entrainment. AIAA J. 25, 578-583.

Kee, R. J., Millier, J. A. \& Jefferson, T. H. 1980 CHEMKIN : A general purpose, problem independent, transportable, Fortran chemical kinetics code package. SANDIA Rep. SAND808003 .

Keller, J. O. \& DaILY, J. W. 1983 The effect of large heat release on a two-dimensional mixing layer. AIAA Paper 83-0487 21st Aerospace Sciences Meeting, January 1983.

Kollman, W. \& Janicka, J. 1982 The probability density function of a passive scalar in turbulent shear flows. Phys. Fluids 25, 1755-1769.

KonRaD, J. H. 1976 An experimental investigation of mixing in two-dimensional turbulent shear flows with applications to diffusion-limited chemical reactions. Ph.D. dissertation, California Institute of Technology.

Koochesfahani, M. M., Catherasoo, C. J., Dimotakis, P. E., Gharib, M. \& Lang, D. B. 1979 Two-point LDV measurements in a plane mixing layer. AIAA J. 17, 1347-1351.

Koochesfahani, M. M. \& Dimotakis, P. E. 1986 Mixing and chemical reactions in a turbulent liquid mixing layer. J. Fluid Mech. 170, 83-112.

Koop, C. G. \& Browand, F. K. 1979 Instability and turbulence in a stratified fluid with shear. J. Fluid Mech. 93, 135-159.

LANG, D. B. 1985 Laser Doppler velocity and vorticity measurements in turbulent shear layers. Ph.D. thesis, California Institute of Technology.

Marble, F. E. \& Broadwell, J. E. 1977 The coherent flame model for turbulent chemical reactions. Project SQUID Tech. Rep. TRW-9-PU.

Masutani, S. M. \& Bowman, C. T. 1986 The structure of a chemically reacting plane mixing layer. J. Fluid Mech. 172, 93-126.

McMurtry, P. A., Jou, W.-H., Riley, J. J. \& Metcalfe, R. W. 1986 Direct numerical simulations of a reacting mixing layer with chemical heat release. AIAA J. 24, 962-970.

MoMuntry, P. A. \& RiLEY, J. J. 1987 Mechanisms by which heat release affects the flow field in a chemically reacting, turbulent mixing layer. AIAA Paper 87-0131, 25th Aerospace Sciences Meeting, January 1987.

Mungal, M. G. \& Drmotakis, P. E. 1984 Mixing and combustion with low heat release in a turbulent shear layer. $J$. Fluid Mech. 148, 349-382.

Mungal, M. G., Hermanson, J. C. \& Dimotakis, P. E. 1985 Reynolds number effects on mixing and combustion in a reacting shear layer. AIAA J. 23, 1418-1423.

Mungal, M. G. \& Frieler, C. E. 1985 The effects of Damköhler number on a turbulent shear layer - experimental results. GALCIT Rep. FM85-01.

Paranthoen, P., Lecordier, J. D. \& Petit, C. 1982 Influence of dust contamination on frequency response of wire resistance thermometers. DISA Information 27, 36-37.

Pitz, R. W. \& DaILY, J. W. 1983 Combustion in a turbulent mixing layer formed at a rearwardfacing step. AIAA J. 21, 1565-1570.

POPE, S. B. 1981 A Monte Carlo method for the PDF equations of turbulent reactive flow. Combust. Sci. Tech. 25, 159-174.

Prandtl, L, 1925 Bericht über Untersuchungen zur ausgebildeten Turbulenz, Zr. angew. Math. Mech. 5, 136-139.

Rajagopalan, S. \& Antonia, R. A. 1981 Properties of the large structure in a slightly heated turbulent mixing layer of a plane jet. J. Fluid Mech. 105, 261-281.

Rebollo, M. R. 1973 Analytical and experimental investigation of a turbulent mixing layer of different gases in a pressure gradient. Ph.D. thesis, California Institute of Technology. 
SCADron, M. D. \& Warshawsky, I. 1952 Experimental determination of time constants and Nusselt numbers for bare-wire thermocouples in high-velocity air streams and analytic approximation of conduction and radiation errors. NACA Tech Note 2599.

SPaLding, D. B. 1986 The two-fluid model of turbulence applied to combustion phenomena. AIAA J. 24, 876-884.

SPENCER, B. W. \& JoNES, B. G. 1971 Statistical investigation of pressure and velocity fields in the turbulent two stream mixing layer. AIAA Paper 71-613, 11th Aerospace Sciences Meeting, January 1971.

StÅRner, S. H. \& BiLGER, R. W. 1980 LDA measurements in a turbulent diffusion flame with axial pressure gradient. Combust. Sci. Tech. 21, 259-276.

WALLACE, A. K. 1981 Experimental investigation of the effects of chemical heat release in the reacting turbulent plane shear layer. Ph.D. thesis, The University of Adelaide; also AFOSR Rep. TR-84-0650.

Winant, C. D. \& Browand, F. K. 1974 Vortex pairing: the mechanism of turbulent mixinglayer growth at moderate Reynolds number. J. Fluid Mech. 63, 237-255. 